San Jose State University

SJSU ScholarWorks

Master's Theses

Master's Theses and Graduate Research

1989

\title{
The Effects of types of resource demands on performance of a three-axis tracking task in a dual task paradigm
}

Teresa A. King

San Jose State University

Follow this and additional works at: https://scholarworks.sjsu.edu/etd_theses

\section{Recommended Citation}

King, Teresa A., "The Effects of types of resource demands on performance of a three-axis tracking task in a dual task paradigm" (1989). Master's Theses. 3151.

DOI: https://doi.org/10.31979/etd.fdsp-gjk3

https://scholarworks.sjsu.edu/etd_theses/3151

This Thesis is brought to you for free and open access by the Master's Theses and Graduate Research at SJSU ScholarWorks. It has been accepted for inclusion in Master's Theses by an authorized administrator of SJSU ScholarWorks. For more information, please contact scholarworks@sjsu.edu. 


\section{INFORMATION TO USERS}

The most advanced technology has been used to photograph and reproduce this manuscript from the microfilm master. UMI films the text directly from the original or copy submitted. Thus, some thesis and dissertation copies are in typewriter face, while others may be from any type of computer printer.

The quality of this reproduction is dependent upon the quality of the copy submitted. Broken or indistinct print, colored or poor quality illustrations and photographs, print bleedthrough, substandard margins, and improper alignment can adversely affect reproduction.

In the unlikely event that the author did not send UMI a complete manuscript and there are missing pages, these will be noted. Also, if unauthorized copyright material had to be removed, a note will indicate the deletion.

Oversize materials (e.g., maps, drawings, charts) are reproduced by sectioning the original, beginning at the upper left-hand corner and continuing from left to right in equal sections with small overlaps. Each original is also photographed in one exposure and is included in reduced form at the back of the book. These are also available as one exposure on a standard $35 \mathrm{~mm}$ slide or as a $17^{\prime \prime} \times 23^{\prime \prime}$ black and white photographic print for an additional charge.

Photographs included in the original manuscript have been reproduced xerographically in this copy. Higher quality $6^{\prime \prime} \times 9^{\prime \prime}$ black and white photographic prints are available for any photographs or illustrations appearing in this copy for an additional charge. Contact UMI directly to order.

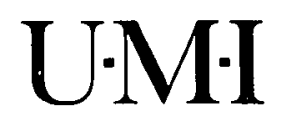

University Microfilms International

A Bell \& Howell Information Company 300 North Zeeb Road. Ann Arbor, MI 48106-1346 USA 


\section{•}


The effects of types of resource demands on performance of a three-axis tracking task in a dual task paradigm

King, Teresa A., M.S.

San Jose State University, 1989 



\title{
THE EFFECTS OF TYPES OF RESOURCE DEMANDS ON PERFORMANCE OF A THREE-AXIS TRACKING TASK IN A DUAL TASK PARADIGM
}

\author{
A Thesis \\ Presented to \\ the Faculty of the Department of Psychology \\ San Jose State University
}

In Partial Fulfillment

of the Requirements for the Degree

Master of Science

by

Teresa A. King

August, 1989 
APPROVED FOR THE DEPARTMENT OF PSYCHOLOGY Pain Veblen

Professor David Weckler, PhD., Chairperson

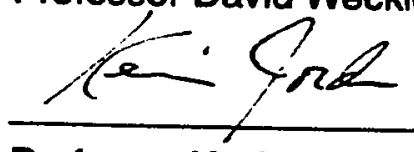

Professor Kevin Jordan, PhD.

Stomerman lloternoto

Joy Hamerman Matsumoto, M.A.

APPROVED FOR THE UNIVERSITY

Serena Mt Peanfore 


\section{Acknowledgments}

I would like to take this opportunity to express my gratitude for the patience, advice, guidance, and encouragement I have received from my family, friends, and professors who have helped make this achievement possible. My advisors, Dr. David Weckler and Dr. Kevin Jordan, and my co-workers have been extremely supportive and I thank them. My children, Katina and Stephen had to spend many evenings "fending for themselves" so that their mother could study and work on her thesis, without them it might have been easier, with them it has been more meaningtul. I would also llke to thank my parents for the emotional support needed to achieve this overwhelming endeavor.

Many thanks to Sandra Hart who seemed to be thwarting my progress with her and Joy's meticulous preliminary work and saying "trust me", claiming it would pay off in the end. I trusted her, and it did pay off.

I would also like to thank the Directors and personnel of the NASA Ames Research Center, Aerospace Human Factors Division for the use of their facilities and services. I would like to acknowledge the support offered by the National Aeronautics and Space Administration Grant NCC 2-327 to San Jose State University Foundation.

Mike Bortolussi and Lowell Staveland deserve special mention. Lowell for his hard work In setting up the style sheets in the Ventura desk top publisher needed to meet the APA format standards for this thesis and Mike Bortolussi who was willing to patiently answer my "quick" questions as I learned Ventura.

Last but not least, I would like to express my deepest gratitude to a very special woman, Joy Matsumoto, who not only served as my most important committee member, but as a teacher, mentor, role model, and friend. 
Table of Contents

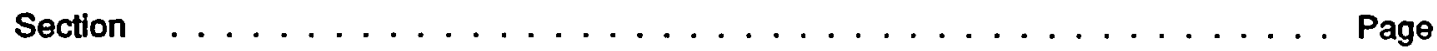

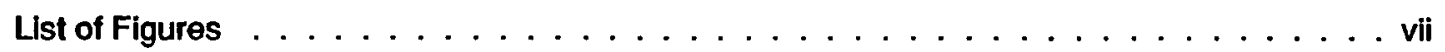

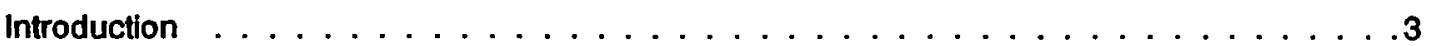

Theories of Information Processing and Attention $\ldots \ldots \ldots \ldots$

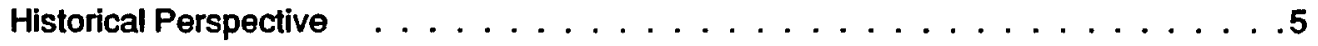

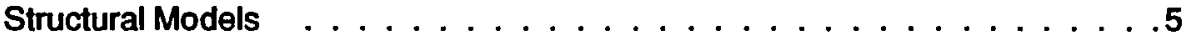

Capacity Models . . . . . . . . . . . . . . . . . 11

Concurrent Task Performance and Workload . . . . . . . . . . . . 19

The Present study . . . . . . . . . . . . . . . . . 22

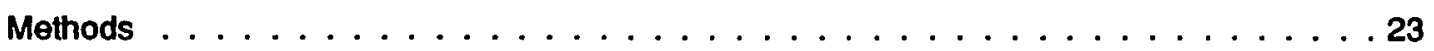

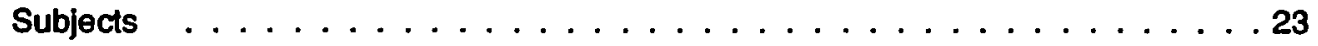

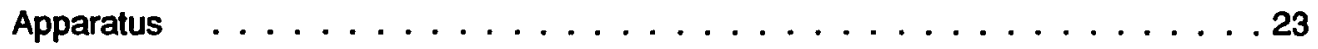

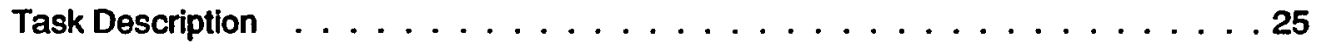

Discrete Tasks . . . . . . . . . . . . . . . 28

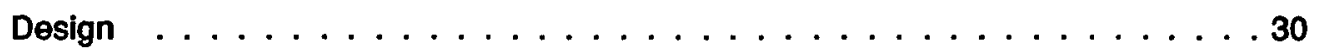

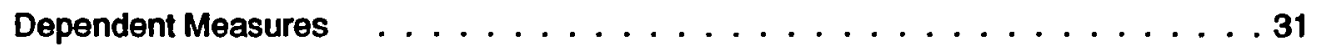

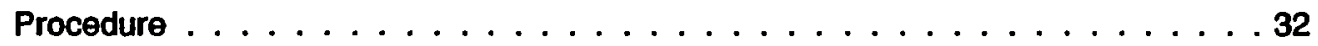

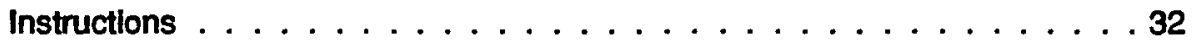

Script Format . . . . . . . . . . . . . . . 32

Practice Trials $\ldots \ldots \ldots \ldots \ldots \ldots \ldots \ldots \ldots \ldots \ldots \ldots \ldots \ldots \ldots$

Experimental Trials $\ldots \ldots \ldots \ldots \ldots$

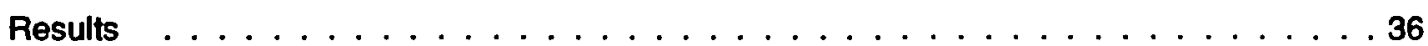


Discrete Task Analysis . . . . . . . . . . . . . . . . . 37

Reaction Time. . . . . . . . . . . . . . . . . . . 37

Percentage of Error . . . . . . . . . . . . . . . . . 41

Component Workload Ratings of the Discrete Tasks $\ldots \ldots \ldots 4$

Tracking Performance Data $\ldots \ldots \ldots \ldots$. . . . . . . . . . . . .

Heading RMSE in Dual Task Condition $\ldots \ldots \ldots 4 . \ldots \ldots$

Altitude RMSE in Dual Task Condition $\ldots \ldots \ldots . \ldots \ldots$

Spe日d RMSE in Duai Tracking $\quad \ldots \ldots \ldots \ldots \ldots$. . . . . . . . . . . .

Component Workload Ratings of the Tracking Axes $\ldots \ldots \ldots 5$

Single versus Dual Tracking Performance $\ldots \ldots \ldots 5$

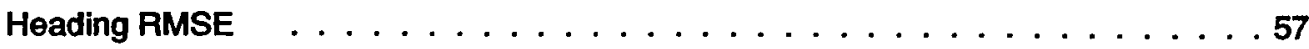

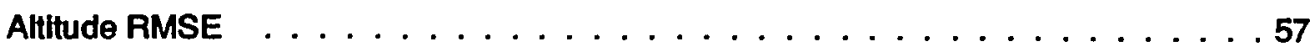

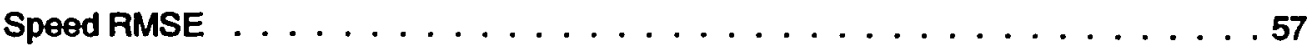

Component Workload Ratings of Tracking $\ldots \ldots \ldots \ldots$

Overall Workload Ratings for the Complex Task $\ldots \ldots \ldots 58$

Discrete Task Condition $\ldots \ldots \ldots \ldots \ldots \ldots$

Single versus Dual oWL $\ldots \ldots \ldots \ldots \ldots$

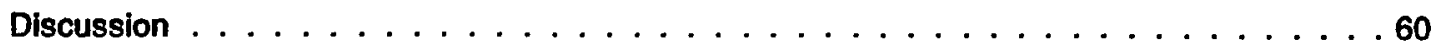

Performance on the Discrete and Tracking Task $\ldots \ldots \ldots \ldots$

Component and Overall Workload Ratings $\ldots \ldots \ldots 66 \ldots \ldots$

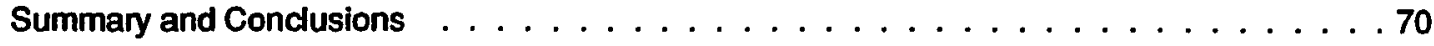

Suggestions for Future Research $\ldots \ldots \ldots \ldots \ldots \ldots$

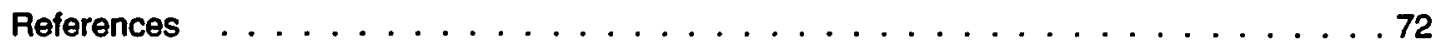




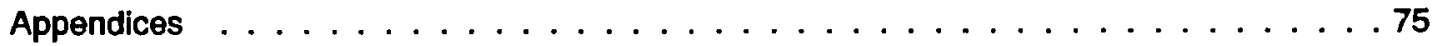

Appendix A .......................

Appendix B ............................

Appendix c .........................

Appendix D . . . . . . . . . . . . . . . 82

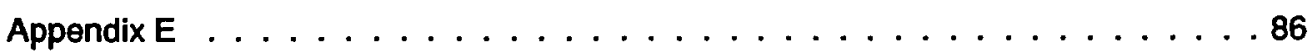




\section{List of Figures}

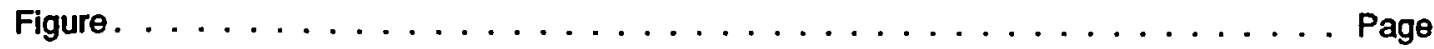

Figure 1. . . Information-flow diagram for Broadbent's fitter theory. . . . . . . . . .7

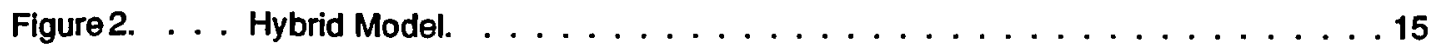

Figure 3. . . The proposed structure of processing resources. $\ldots \ldots \ldots \ldots$

Figure $4 . \ldots$ Subjects' reponse box. $\ldots \ldots \ldots \ldots \ldots \ldots \ldots$. . . . . . . . . .

Figure 5. . Example of the monitor display. . . . . . . . . . . 26

Figure6. . Examples of the CRT task. . . . . . . . . . . . . 29

Figure 7. . . Reaction time as a function of difficulty and resource. . . . . . . . . . . 39

Figure 8. . . Reaction time as a function of difficulty and task. . . . . . . . 40

Figure 9. . . Reaction time as a function of difficulty, resource and tracking presentation. . .42

Figure 10. . Reaction time as a function of difficulty, resource and task. . . . . . . 43

Figure 11. . Error rate as a function of difficulty and task. $\ldots \ldots \ldots \ldots$

Figure 12. . Error rate as a function of resource and task. . . . . . . . . 46

Figure 13. . Error rate as a function of tracking presentation and task. . . . . . 48

Figure 14. . Heading RMSE as a function of axis changes. . . . . . . . . . . 50

Figure 15. . Heading RMSE as a function of noise and change in axis. . . . . . . 51

Figure 16. . A Altitude RMSE as a function of axis change. . . . . . . . . . . . 53

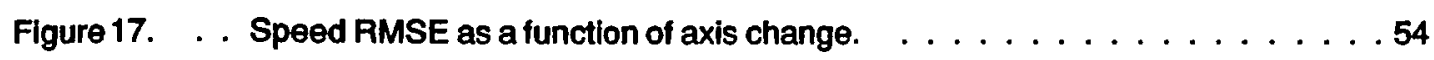

Figure 18. . . Speed RMSE as a function of discrete task difficulty and tracking difficulty. . . 56

Figure 19. . . Workload as a function of the discrete task difficulty and tracking presentation. 60 
Dual Task Performance

THE EFFECTS OF TYPES OF RESOURCE DEMANDS ON PERFORMANCE OF A THREE-AXIS TRACKING

TASK IN A DUAL TASK PARADIGM

TeresaA. King

San Jose State University

Running head: Dual Task Performance

Requests for reprints should be sent to Teresa A. King, Department of Psychology, San Jose State University, San Jose, California 95192 
Dual Task Performance

\begin{abstract}
This study investigated four factors which may affect performance and workload in a dual task paradigm. These four factors, associated with attention theory, are: the use of different central processing codes (i.e., verbal or spatial resources based on the multiple resource capacity model); the difficulty levels of each component task; and the effect of presenting both tasks simultaneously. Contrary to the predictions of the multiple resource model, performance was not differentially affected by the verbal or spatial discrete task. It was expected that as the difficulty level of the spatial discrete task increased, performance on the continuous task would decrement. Continuous task performance did not decline as the difficulty of the discrete tasks increased; nor was there a significant effect on the discrete task performance as a result of the difficulty manipulation on the continuous task. This could indicate that subjects were willing to work harder and therefore increase their available resources through additional effort, or that there is no competition for the same resources, but rather a limited amount of resources in general.
\end{abstract}




\section{The Effects of Types of Resource Demands \\ On Performance of a Three-axis Tracking Task in a Dual Task Paradigm}

The simultaneous performance of more than one task is a common phenomenon. When performing several tasks it is not unusual to experience demands on more than one mental or physical resource. We have little understanding of how concurrent task activities are processed, or the effect on workload and individual task performance during the execution of two simultaneous tasks? To address these issues, it is necessary to better understand existing theories of divicied attention and the limitations these theories may impose on central information processing.

One approach to understanding the limitations imposed on information processing and its impact on workload during the execution of concurrent tasks is to compare measures of workload and task performance during the execution of concurrent tasks to the same measures of the same tasks performed individually. This study investigated four factors which may affect performance and workload in a dual task paradigm. These four factors, commonly associated with attention theory, are: the use of different central processing codes (i.e., verbal or spatial codes); the difficulty level of the first task; the difficulty level of the second task; and the effect of presenting both tasks simultaneously as opposed to presenting the tasks individually.

This thesis discusses the historical progression of information processing and attention theory research as related to performance and workload in a dual task paradigm. Based on the multiple resource model the specific purpose of this study was to test the various information processing theories by investigating differences in periormance and workload obtained during different combinations of task presentations. The definition and the dimensions of different 
resources, specifically verbal and spatial, were addressed. The effects of combining two tasks and of increasing the difficulty level of each task was explored by comparing single-to-dual task performance and workload at the two levels of difficulty. Subjective ratings of overall workload of the entire dual task condition were compared to workload ratings of the components (single discrete tasks and three axis of a continuous tracking task) of the dual task and to workload ratings of single tasks performed alone. This was done in order to determine i: subjects were able to consistently rate subjective workload for a component of a task when it was paired with other components or when it was performed alone.

\section{Theories of Information Processing and Attention}

The processing of information is dependent on the characteristics of the task as well as the environment in which the task is performed. There are many different models concerned with the processing of information under multi-task conditions. Two general models, structural and capacity, attempt to account for the variance in the efficiency of time- sharing.

Structural models contend that interference in the performance of two tasks executed simultaneously is due to the competition for common channels or mechanisms in the input stage (Treisman, 1964a; Broadbent, 1958). According to Treisman (1964a), the word channel refers to a "discrete physical system with defined properties" (p. 13). These propenties may describe sense organs such as the eye or ear. Using auditory stimuli, Treisman (1964b) found that pitch and semantic message content could both define a channel.

Capacity models contend that limitations of attention are due to there being a set amount of central processing codes which are available to process input. These models state that interference occurs in more than just the input stage; capacity models address the modality of 
output, as well as the central processing or cognitive processing stages as sources of interference (Wickens, 1984b).

Historical Perspective

Historically, the juxtaposition of attention theory and information processing began with Broadbent in the early 1950's (e.g., Broadbent, 1958). One of the important issues has been multi-task interference, which seemed to occur at different times and under different conditions. The hypothesis of information processing interference developed into research focusing on bottlenecks of information and where such bottlenecks occurred. The concept of a bottleneck refers to the point at which the amount of information to be processed exceeds the capacity of the system to process it effectively. An active line of research stemming from the bottleneck research has been the aftempt to account for the variance in dual task performance.

Structural Models. Broadbent (1958) pioneered the concept of a limited capacity channel model, and the idea that an overload of information would become bottlenecked and not processed effectively. He included the construct of a selectlve filter before the actual central processing of information. Broadbent theorized the bottleneck of information occurred at the stage of perceptual analysis since only information about one stimulus at a time can be passed through the selectlve filter.

Broadbent's (1958) filter theory was one of the first early selection models developed. His three-step process, which occurs before central processing, assumes a sequence of three elements involving sensory input: (a) short-term store (S-system); (b) a selective fitter; and (c) a limited capacity channel where perceptual analysis takes place (P-system). Information enters the system through a number of parallel sensory channels where it is registered in the S-system. The channels are set up to separate incoming sensory information by the general physical 
characteristics of the stimuli. The P-system has a limit to its capacity; the S-system does not have a limited capacity. At the next stage of input evaluation, the selective filter allows only certain stimuli through to the P-system. Since Broadbent assumed parallel processing did not occur within the P-system, only information regarding one stimulus is passed through the filter at any time. Which information is passed through the system depends on the personality, mood, or state of the organism as well as the salience of the stimuli. If two stimuli are presented at the same time, the sensory input from one will be perceptually processed while the other is held in the S-system. This input will stay in the S-system until: (a) the selective filter allows it to go on to the P-system for analysis, (b) the information decays, or (c) it is replaced by new information. The amount of time spent analyzing sensory information in the P-system depends on the extent of information contained by the stimulus.

When attention is focused the filter is set to allow only information pertaining to the specific activity being performed to enter the P-system. Therefore, attention is most effectively focused when the sensory input can clearly be distinguished by its general physical characteristics in contrast to semantic characteristics. In other words, it is easier to divide attention between two dissimilar tasks such as listening and looking, where the inputs are quite physically distinct, than between two similar tasks such as listening to two different voices, where the distinction between inputs is primarily semantic. According to Broadbent (1958), the apparent division of attention in the performance of two concurrent activities can be explained by the alternation between channels (such as left and right ear, or different pitch as well as ilstening and looking). The rate of alternation is slow, approximately 300 to $500 \mathrm{msec}$, and since only one stimulus at a time can be analyzed, the bottleneck in this model occurs at the stage of perceptual analysis (see Figure1). 


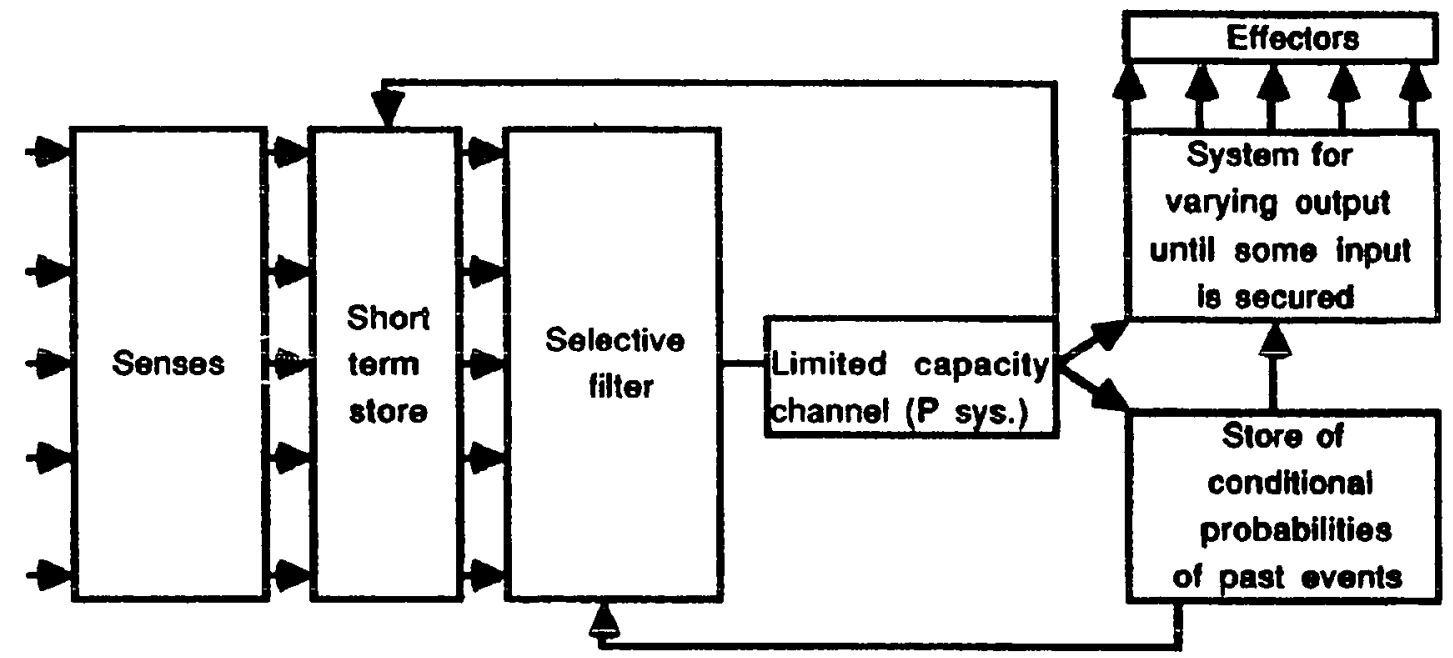

Figure 1. Information-flow diagram for Broadbent's filter theory.

(From Broadbent, 1958) 
Early studies such as Cherry (1953), Moray (1959), and Treisman (1960, 1964b) confirmed that attention is limited, but questioned Broadbent's (1958) hypothesis concerning the point at which a bottleneck or overload in information processing might occur. They also disagreed with Broadbent's definition of the filter. The discussion pertaining to the location of the bottleneck is important when discussing theories of attention since the goal is to understand how to overcome the bottleneck for enhanced information processing and minimal interference.

Cherry (1953) conducted a series of experiments investigating subjects' abilities to shadow two concurrent messages. He presented two different passages of prose, one to each ear, and asked subjects to shadow one of the passages. Cherry's findings demonstrated that subjects could repeat back the passage to which thyy were attending but were unable to repeat any of the prose presented to the other ear. Subjects could, however, distinguish certain acoustical properties such as the frequency spectrum or intensity. Subjects could not relate any of the meaning of the two messages nor did they detect a change from English to German in the non-shadowing ear. Cherry concluded that except for some general characteristics, subjects were able to block one channel of irrelevant information and therefore were somewhat able to avoid the bottleneck at the perceptual stage.

Moray (1959) found that his subjects, like Cherry's, could effectively block the contents of a rejected message. Moray's subjects were asked to judge the suitability of words in a passage. Three lists of seven words were used; the first list actually occurred in a passage, while the other two lists did not. All words from the three lists were equally appropriate for the context of the passage. Subjects were presented with the passage to be shadowed in one ear and one of the two lists with words not in the passage were presented in the other ear. After shadowing the passage subjects were asked which words they heard during the shadowing passage. The 
results supported Cherry's (1953) finding; subjects demonstrated they could block information by failing to recognize any of the material from the two irrelevant lists.

Treisman (1964a, 1969) proposed a modification of Broadbent's (1958) theory that information enters the processing system through a single channel and some information simply does not get through the filter. Instead, she theorized that information enters the general processing system through a number of channels. As this information reaches the selective filter, the channels are analyzed by their physical (i.e., location or tone) or semantic characteristics (i.e., meaningfulness of the word). Each analyzer functions as a single channel and different analyzers can operate in parallel without interference, although processing within a single analyzer is serial. With the analyzers working in parallel, Treisman believed that an attenuation (of information input) process occurs. Through attenuation all information may be processed but is not necessarily attended to. Thus the more salient the information the less selective blocking occurs.

In order to test the model described above. Treisman (1971) investigated the effect on periormance of attending to more than one irrelevant message at a time while shadowing. The variables manipulated were: (a) the number of irrelevant messages $(0,1$, or 2$)$; (b) irrelevant channels (separate messages were presented aurally in each ear or simultaneously to both ears) and; (c) the spatial localization (in each ear separately or the center of the head) of the irrelevant messages. The subjects were required to shadow one message in the presence of 0,1, or 2 Irrelevant messages presented simultaneously. Treisman's findings indicated that presenting one irrelevant message on a separate channel did not affect shadowing performance. However, adding two irrelevant messages on two separate channels did affect shadowing. There was a decrement in performance while three channels were stimulated but not when two irrelevant 
messages were presented on the same channel. Treisman's results suggested that selective blocking or attenuation occurred, which she believed was a different process from the analysis of signals into different channels. In other words, Treisman felt that the information was processed, but not attended to. Broadbent's filter theory did not allow for certain information to pass through it; thus he concluded that the information is not even available for processing.

Treisman continued with follow-up studies of the 1964(b) experiment. In 1970 she demonstrated that parallel processing is possible with verbal stimuli. Treisman's subjects were presented auditory stimuli with either a single item or synchronized pairs of items consisting of two nonsense syllables or one nonsense syllable and one digit. The subjects were to press a particular key if a digit was present and another if no digit was present. Subjects were pre-cued (i.e., presented a clue as to which digit was going to be present) on some trials about digit information. It was presumed subjects would test the input presented to one ear. If it did not contain a digit, they would switch to the other ear and test for digit information. If subjects were pre-cued it was thought that a larger reduction in reaction time would occur with pairs than with single presentations as pre-cuing would cut out a stage of processing. Surprisingly, the results showed that pre-cuing decreased reaction time by $115 \mathrm{~ms}$ in both the single and paired presentations. The implication was that subjects can process information in paraliel. Treisman (1970) predicted subjects should be able to monitor two messages as easily as one, provided the messages reach two separate analyzers. Treisman and Davies (1972) tested her 1970 theory by presenting the incoming messages in two different modalities, visual and/or auditory. Subjects were presented with pairs of auditory and visual stimuli so that the input modalities were either the same (visual/visual or auditory/auditory) or different (visual/auditory or auditory/visual). The required task was to perform channel by channel recall, always beginning with the left ear, 
mimicking the input information, which included: repeating auditory words, singing auditory tones, writing visually presented letters, and marking visual positions. The results indicated that significantly more interterence occurred when the stimuli were presented in the same modality than when they were presented in a visual/audio modality combination. The results of their study supported Treisman's 1964h study in that subjects could monitor two messages as well as one as long as they were not being processed by the same analyzer. By using the same input modality, the same analyzers were being utilized. As mentioned previously, Treisman believed that serial processing occurs within a given analyzer, but in different analyzers, parallel processing can occur.

In summary, early selection structural models distinguish physical from semantic attributes of information. The information then proceeds through different channels before it reaches central processing (Broadbent, 1958). Later structural models claim that different channels may be processed in parallel, but within each channel the information is processed serially (Treisman, 1964a, 1969). The models of multi-channel processing postulate a limited number of channels.

Capacity Models. Capacity models do not deny all aspects of the structural models, but rather elaborate on them by discussing the limiting factor of information processing from a different perspective and add that interference may occur in the central processing stage (Wickens, 1980; Navon \& Gopher, 1979). Capacity models claim a limilation of resources, but allow for those resources to be refueled under certain conditions (Kahneman, 1973).

Capacity models propose that people possess a pool or pools of resources. Given the assumption that there is an undifferentiated limited pool of resources, performance on one task will deteriorate when the demands of a second task increase (Kantowitz, 1988). This is assumed to be due to fewer resources being available to process the second task. In general, interference 
between the tasks is assumed to be due to a limit of, or unavailability of resources. According to Norman and Bobrow (1975), a resource is a theoretical construct which includes aspects such as effort for the processing of information, various forms of memory capacity, and the communication channels.

While Broadbent believed the process of attending to more than one task at a time is accomplished through alternation, Allport, Antonis, and Reynolds (1972) have shown that two concurrent tasks may be performed in parallel. Subjects were asked to play the piano (while sight reading the music) with or without concurrent shadowing of prose. In the dual task condition neither of the tasks was designated as the primary task. There were two grades of music and two levels of difficulty for the prose. The results showed that there was little or no loss in efficiency in piano playing or shadowing when both tasks were performed concurrently. As a result of this finding, Allport et al. (1972) developed a multi-channel hypothesis which produced the allocatable capacity model. One of the major criticisms of this study has been the use of piano playing by experts; critics feel the piano playing task was overlearned thus rendering conclusions based on the performance of the piano playing task suspect.

As research in attention theory progressed, other studies (Kantowitz \& Knight, 1976a; Navon \& Gopher, 1979; Vidulich \& Wickens, 1981) have shown that Broadbent's (1958) filter theory and single channel theory (Kinsboume, 1981) are inadequate in explaining results supporting parallel processing (Treisman \& Davies, 1972; Ninio \& Kahneman, 1974; McLeod, 1977).

As a result of his previous work (Moray \& Jordan, 1966), Moray $(1967,1969)$ proposed that a central processor of limited capacity receives, transforms, and produces messages. The task or task set determines where the channel is narrowest, and if any spare capacity is available for 
other tasks. Moray agreed with the Deutsch and Deutsch (1963) model of a single output channel. As a result of this Moray believed some parallel to serial processing must take place. According to Moray (1969), in contrast to Treisman (1969), a delay in output or response is due to the transformation of parallel to serial processing rather than switching between different channels. Moray and Jordan (1966) found that compatibility between the output and input (i.e., same modality) of information eliminates the need for transformation: therefore, a processing function (transformation) is omitted and information processing capacity is increased.

Kahneman (1973) proposed a variable allocation model of attention. This model describes a central pool of resources which varies according to the state and circumstances of the individual. The evaluation of demands upon the system is controlled by a central system which, In accordance with an allocation policy, distributes the resources. According to Kahneman, the allocation policy is controlled by the following: (a) involuntary attention (e.g., allocation of resources to a novel stimulus such as a personal introduction); (b) immediate objectives (e.g., looking for a police car while speeding); (c) assessment of demands (when two activities demand more resources than are available, only one is completed); and (d) systematic changes in allocation due to arousal states.

Kahneman (1973) proposed that attention is selective and controllable and is dependent on both the permanent dispositions and the temporary goals or intentions of the individual. His variable allocation model centers on the arousal state and degree of effort the individual is willing to expend, which are not determined until the task presents itself. Kahneman, unlike other researchers in attention, places a heavy emphasis on the effects of effort on attention for the amount of capacity available. Thus the available capacity is determined by effort and arousal. 
Further research in attention theory related to the capacity model was performed by Kantowitz and Knight (1974, 1976a). They proposed a hybrid model with a combination of serial and parallel information processing (see Figure 2). Kantowitz's model is based on the assumption that there is one central pool of resources with satellite structures which borrow from each other, but cannot exceed its set central pool capacity. This model proposes a limited capacity where the resources are divided between the stimulus input and response output demands. The more mental activity required to perform a task the greater the demand will be for processing resources. Mental activity generally increases as the number or difficulty of tasks increases.

This hybrid model evolved through a series of experiments. In a pair of experiments subjects were asked to perform a tapping task (low-rate and high-rate conditions) and a digit-naming task with four separate levels of difficulty: naming the digit which appeared, subtracting a digit, adding three to the digits, or subtracting nine from the digits. Separate groups either performed the tapping task or digit-naming task in a single task condition. As difficulty of elther one or both tasks increased, performance decrements occurred in additive stages. Kantowitz and Knight (1976a) concluded that additive stages could co-exist within the limited capacity model. They reasoned that the source of limited capacity feeds both the input and response stages and that these stages may be traded off as long as the capability of the limited capacity source is not exceeded.

Kantowitz and Knight (1976a) state that variable allocation and limited capacity models cannot account for dual-task additivity. Instead they proposed the hybrid model which incorporates parts of a stage model (Kantowitz \& Knight, 1974). A stage model claims that each stage has its own independent source of capacity. The stage model is based on four major 
Serial Stages

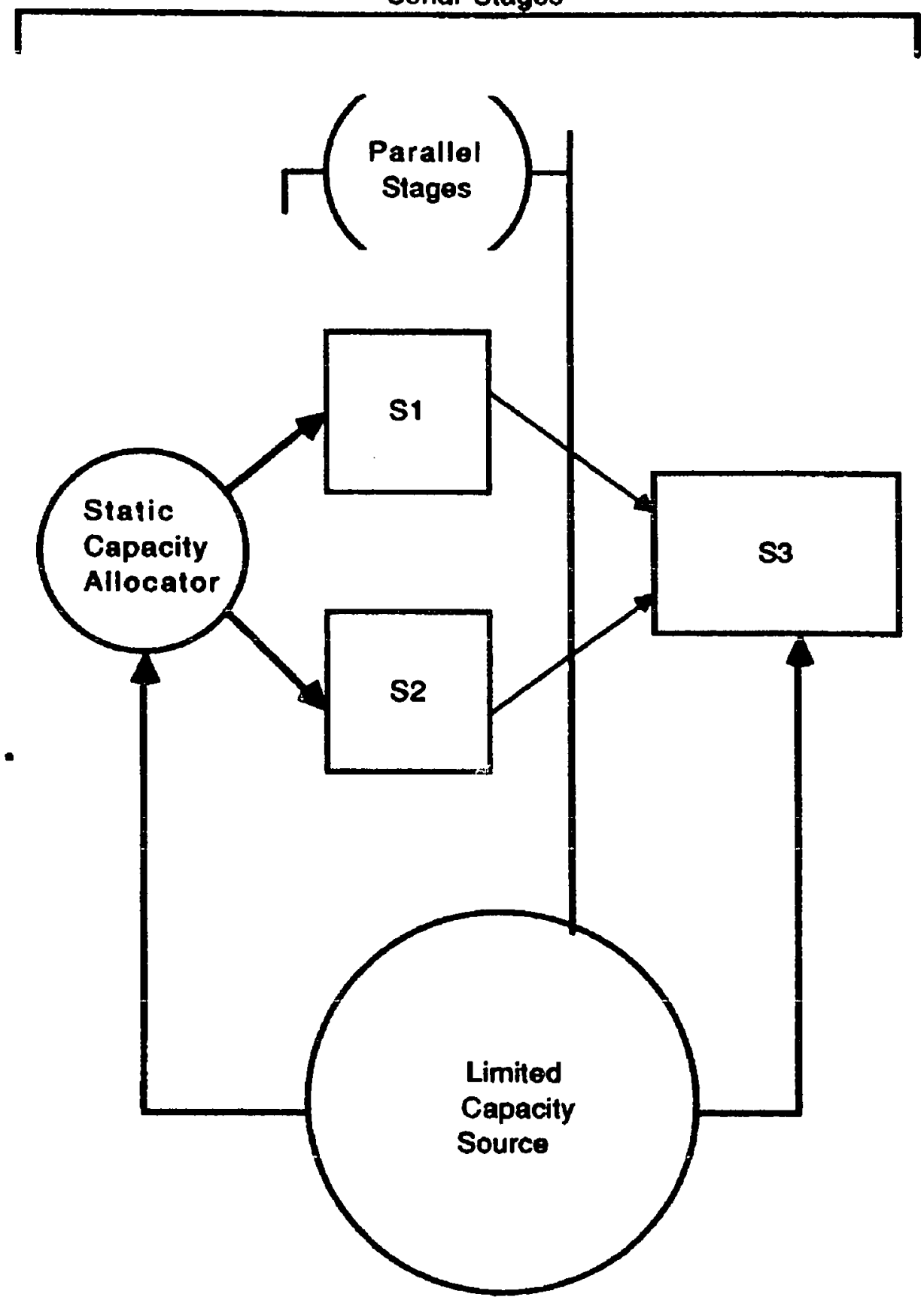

Figure 2. Hybrid model. (From Kantowitz \& Knight, 1976b) 
stages of information processing: stimulus encoding, categorization, response selection, and response execution. This is in contrast to the capacity models which state that there is a common source of capacity. One of these stages could be associated with input processing. Therefore, the division of capacity may be fixed by different situations such as environmental conditions (e.g., incentive pay-offs) or by specific instructions which establish the relative importance of the tasks. They postulate that these stages process in parallel and therefore cannot trade-off capacity with each other because some of the limited capacity is dedicated to the individual processing stages. The processing of these independent stages can produce dual-task additivity. On the other hand, difficulty should also affect efficient processing even though different resources are used if the set capacity limit is reached, whether or not there are separate resource pools (Kantowitz, 1988). This decrement in performance occurs regardless of the stage of processing since demands on the separate stages vary. Wickens (1984a) refutes Kantowitz's hybrid model by stating that if information is processed in separate pools (i.e., different resources are utilized), then different levels of difficulty will have differential effects on performance but the impact will be less, particularly it different resources are demanded, than if there is only one central pool of resources.

The multiple resource model was proposed by Navon and Gopher (1979) and refined by Wickens (1980; see Figure 3). This model states that there is more than one commodity within the processing system which can be given resource-like properties. There are three dimensions that underlie the structure of these resources: stages of processing, input and output modalities, and processing codes.

Two separate resources are defined by processing stages: those related to perceptual-cognitive activity and those associated with the response process (Wickens, 1980; 


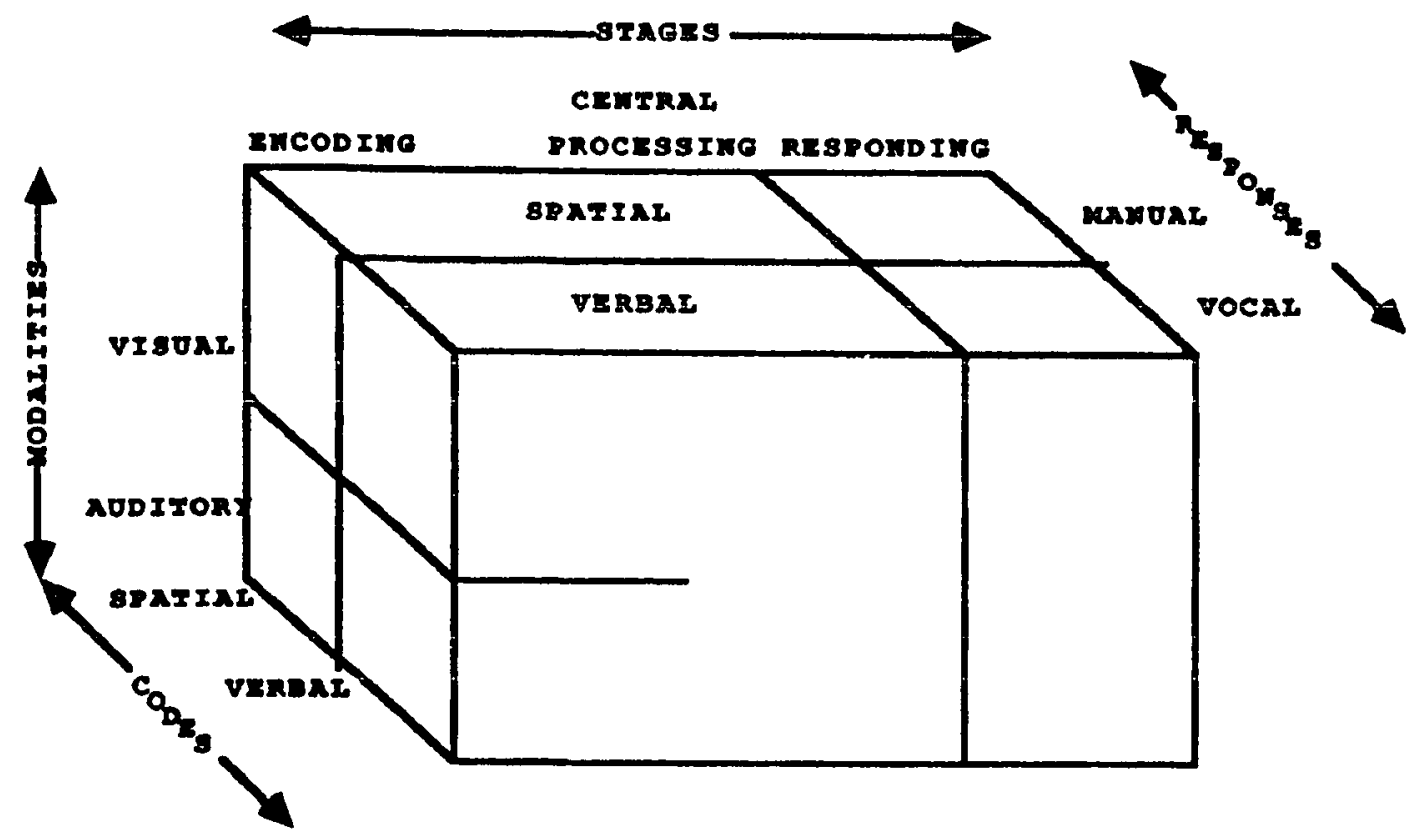

Figure 3. The proposed structure of processing resources.

(Wickens, 1980) 
1984b). Tasks which place demands on perceptual-cognitive processing can be more effectively time-shared with those involving response processes (Wickens \& Liu, 1988). Therefore, if tasks involving perceptual processing are made more difficult, this should have little or no effect upon the performance of tasks demanding response processing. Kantowitz and Knight (1976a) provide support for stage processing as do a number of other researchers (Vidulich \& Wickens, 1981; Wickens, 1980). The second dimension underlying the structure of resources involves input and output modalities. Research has shown that individuals are more capable of dividing their attention between modalities (visual/auditory), than they are within modalities (visual/visual). A study conducted by Boles and Wickens (1983) supported this aspect of multiple resource theory by demonstrating that the less overlap there is between the resource stimulus modalities, the less interference is observed between simultaneous tasks. In their experiment, displays were considered homogeneous if two stimuli indicating a numerical value were presented in the same (vertical/bar graph [spatial] or horizontal/words [verbal]) orientation. They were considered to be heterogeneous if one stimulus of each orientation was displayed. They found that reaction time and percentage of errors decreased when heterogeneous information displays were presented as opposed to homogeneous displays.

As mentioned eartier, Treisman and Davies (1972) found significantly more interference when stimuli were presented in the same modality than when stimuli were presented in different modalitles during the performance of a channel-by-channel recall task. Their study may be interpreted as supporting capacity models. Other studies (Wickens, 1980; McLeod, 1977) also indicated greater time-sharing efficiency when cross-modality response is used.

The last dimension underlying the resource structures involves processing codes. This dimension separates cognitive and response operations which have a linguistic, symbolic (verbal) 
base from those which have an analog (spatial) base (Wickens \& Liu, 1988). Support for separate resources underlying verbal and spatial central processing can be seen in the study by Allport et al. (1972) in which subjects were able to perfectly time-share their attention between piano playing and shadowing.

According to multiple resource theory, there is less interference in a dual task environment when the two tasks require different central processing resources (e.g., spatial and verbal) than when the resources are the same (spatial and spatial or verbal and verbal). Multiple resource theory proposes that there are several different codes for processing information with different resource properties (Wickens, 1984a and 1984b). Wickens discusses two separate pools of capacity, verbal and spatial, which are used for processing information. Interference occurs when tasks compete for the same resources. Wickens' model is much like Kahneman's (1973) variable allocation model; both state that the limits on the pool of resources vary. In Kahneman's model the resources are undifferentiated, and interference is due to direct competition for a single undifferentiated pool. Wicken's claims the resource pools are differentiated and interference occurs when resources are required from the same pool.

\section{Concurrent Task Performance and Workload}

It has been found that during the simultaneous presentation of two distinct tasks there is usually a performance decrement in elther one or both of the two component tasks provided the processing requirements exceed the available channel capacity (Kantowitz \& Knight, 1974). In order to reach this set capacity, difficulty manipulations of both tasks are important. If the set capacity of avallable resource is not reached, performance on either task may not show a dual task decrement. This could be due to the fact that the sum of the necessary resources is less than the available capacity. 
In a dual task environment as one task increases in difficulty it will produce more interference with the concurrent task. The increasing difficulty of the first task will produce a progressive performance decrement on that task. Many times the extent to which the performance decrements on either task will be dependent on the designation of task importance such as secondary or primary task. The task designated as secondary will suffer the greater performance decrement when both tasks are presented. According to Wickens and Liu (1988) the effect of assigning priorities to the tasks will be dependent on the specific processing resources utilized. The resource model predicts that interference will be greater within a resource than when utilizing different processing resources (i.e., spatial and verbal).

An increase in demand on attentional resources may be dealt with by increasing the mental and physical effort expended on the task so that performance is not decremented. Broadbent (1982) defined workload as the cost of switching from one focus of attention to another or the compettion for attentional mechanisms, but his definition may not reflect additional effort (Kahneman, 1973) or motivation (Wickens \& Yeh, 1986). Workload differences may be misrepresented in performance data since performance measures do not always reflect subjective workload assessments. There may be specific cognitive processes responsible for subjective assessments which can differ from those responsible for performance (Vidulich \& Wickens, 1986). Vidulich and Wickens made the assumption that automaticlty is not represented in the consciousness and has little influence on subjective workload, since workload assessments are a conscious cognitive process. Using this assumption in addition to the concept that increased motivation improves performance, the Vidulich and Wickens study used a continuous tracking task and a discrete Sternberg memory set task to test the conditions in which these two different types of cognitive processing, referred to as dissociation, may occur when rating 
workload and performance. Vidulich and Wickens found dissociation occurred when the inter-stimulus-interval was reduced; reaction time increased but subjective workload decreased. In other words, at a high difficulty level, performance was worse but workload ratings were lower. Wickens and Yeh 1988) defined these weak or nonsignifciant relationships between workload ratings and achieved performance as dissociation.

Gopher and Donchin (1986) refer to the interaction between effort and performance as workload. The requirement to perform a secondary task simultaneously with a primary one may result in a more difficult task than a single task particularly if the two tasks do not overlap or integrate in some manner. Gopher and Donchin's (1986) definition of workload suggests that in order to maintain consistent performance on both of these tasks, individuals will increase their effort, thus increasing workload.

Workload ratings are usually collected for predetermined intervals (Hart \& Staveland, 1988) rather than for specific components of a complex task. Workload research has indicated that subjects can provide reliable and meaningful workload ratings for intervals which vary in length and have just occurred, or have occurred in the past. Subjects are adept at integrating or averaging workload imposed by different task elements and in integrating variations in workload over time. According to S. G. Hart (Personal communication, November 1988), there are no reported instances in which workload ratings were specifically directed toward an isolated component or element of a complex task. Although there has been some applied research conducted in which a particular aspect (i.e., mental or physical effect) of a complex task has been isolated and rated, these ratings were obtained for an entire task and focused on particular psychological or physical dimensions as opposed to the workload of a specific component of a complex task (Hart \& Staveland, 1988). 


\section{The Present Study}

The present study addressed some of these issues by measuring subjective workload and performance indicators for two tasks presented both simultaneously and separately. A three-axis pursult tracking task which required spatial resources according to the Wickens (1984b) model, was presented as the continuous task. Two sets of discrete tasks were paired with the continuous tracking task. One set of tasks required verbal central processing and the other required spatial central processing resources. Wickens and Liu (1988), in a research survey, have not found data in which both the codes of processing for the tasks and the task demands (difficulty levels) have been manipulated. They found that contrasts between spatial and verbal processing codes have been confounded by other variables since usually all other aspects of the spatial and verbal conditions are not held constant (i.e., the tasks requiring verbal and spatial processing are not equivalent types of tasks). In order to avoid one of the confounds, Wickens and Llu (1988) feel it is necessary to manipulate the difficulty of both tasks and to maintain equivalence among variables when contrasting spatial and verbal processing. The point of this experiment was to maintain the spatial resource demands of one task (continuous tracking) and manipulate the resource demands of a second discrete task without specifically prioritizing the tasks. This was done to avoid the primary and secondary task distinction. In order to affect the amount of avallable channel capacity (Kantowitz \& Knight, 1976b), the tracking task and the discrete tasks were presented at two levels of difficulty.

To test for ratings consistency, workload ratings were collected on the components of the complex task in varied environments. The single-to-dual task workload components ratings were examined as well as the individual components of the tracking task (heading, speed, and altitude). 
The design of this experiment was based on the multiple resource model. To find support for this model, it was expected that: (a) in the dual task condition the performance of the spatial discrete tasks and/or the continuous spatial tracking task would suffer a greater performance decrement than the verbal discrete tasks and/or tracking task; (b) the increased degree of difficulty of the discrete tasks would cause performance to degrade more when there is a competition for resources as opposed to when there is no competition; (c) dissociation of workload and performance will occur regardless of the resource demands required for the discrete tasks; (d) component workload ratings will be the same in the single task condition as in the dual task condition.

\section{Methods}

\section{Subjects}

Twelve paid male volunteers were recruited from the Bionetics Corporation subject pool at NASA/Ames Research Center. All were right-handed with normal vision, between the ages of 18- 35. Confidentiality of the subjects was maintained by using a coded identifier rather than the subjects' names. The master file of the subjects' names and codes was destroyed at the end of the experiment after the subjects had been paid.

\section{$\underline{\text { Apparatus }}$}

Software for the experimental task was developed at NASA Ames and was presented on an IBM AT computer with a full color monitor. The subjects' response panel consisted of a two-axis joy-stick, one horizontal and one vertical slide potentiometer (slide pot), six buttons, and an enter button (see Figure 4). The joy-stick controlled altitude (up and down movements) and heading (left to right movements) of the subjects' simulated aircraft. The horizontal slide potentiometer was not utlized in this experiment. The vertical slide pot was used to control 


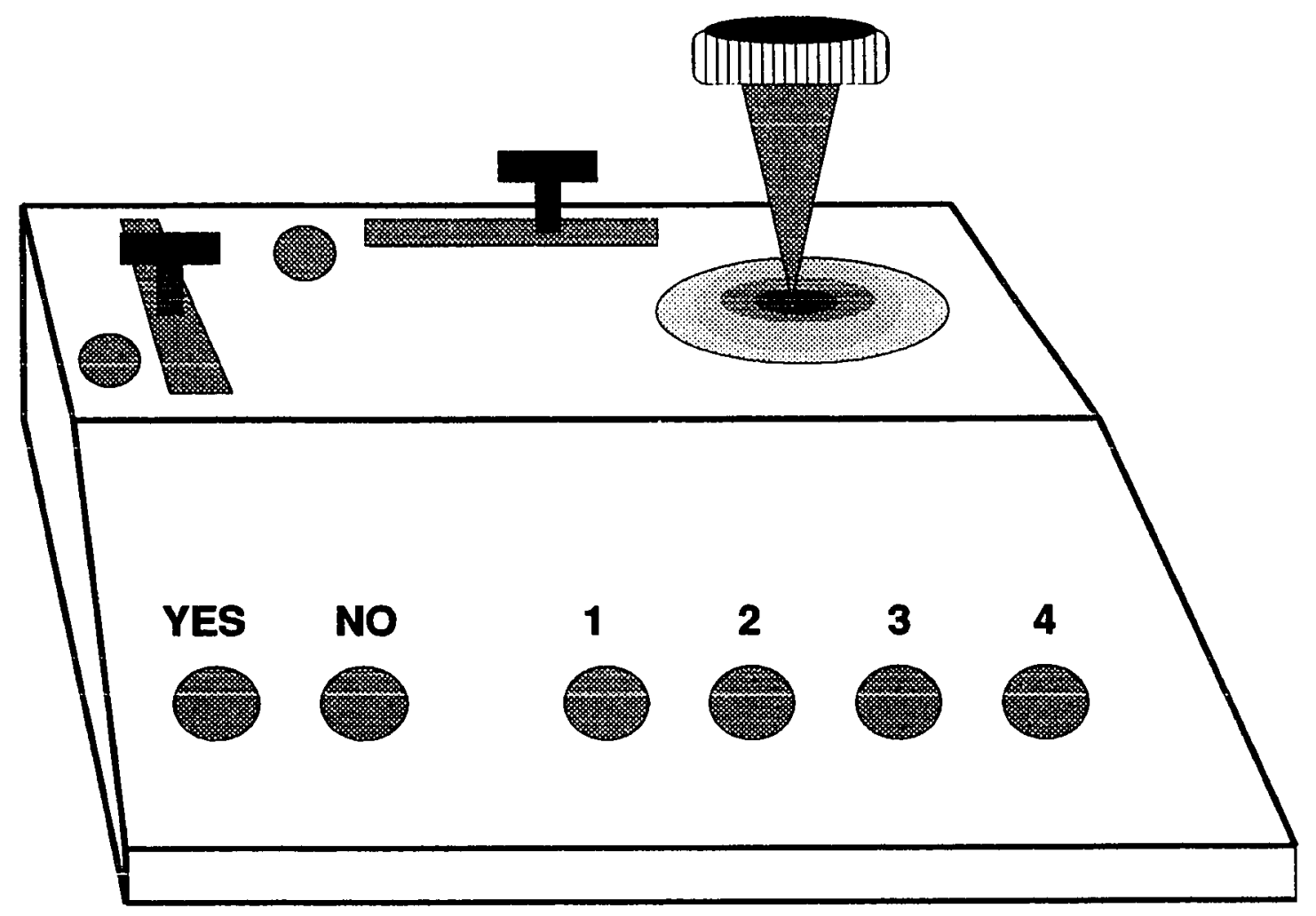

Figure 4. Subjects' response box. 
speed. The bottom of the slide pot represented zero speed and the top represented the maximum speed (160 knots). The buttons were used to respond to the discrete tasks. The two buttons on the left were reserved for "yes" and "no" responses. The four remaining buttons served as position indicators for a four-choice reaction time task. The six buttons were labeled "yes", "no" and "1, 2, 3, 4".

Workload ratings were collected for the components of each task. The basic components of the dual task were speed control, heading control, altitude control, discrete Task 1, and discrete Task 2. The overall rating for the whole complex task was also obtained. When tracking was presented without the discrete tasks, the workload ratings for only the spe日d, heading, and althude control components were collected. When the discrete tasks were presented without tracking the workload component ratings were only collected for each of the two discrete tasks. These multiple component workload scales were obtained in the same format as the workload scale. The scales were presented on an $21.6 \mathrm{~cm}$ by $27.9 \mathrm{~cm}$ sheet of paper and the subjects marked a slash along the continuum at the point which best reflected the subjects' overall workload (OWL) for the given trial (see Appendix A).

\section{Task Description}

Task describes the type of activity or activities the subjects were required to perform. The experiment utilized a software package which presented information in four separate quadrants on a color monitor (see Flgure 5). The primary task was a thre-axis combination pursuit and compensatory tracking task. The subjects were required to fly an aircraft which followed a target represented by a circle (pursuit tracking). A scenario written by the experimenter described the task activities, tracking movements and wind factors (compensatory tracking), along the three axes. 


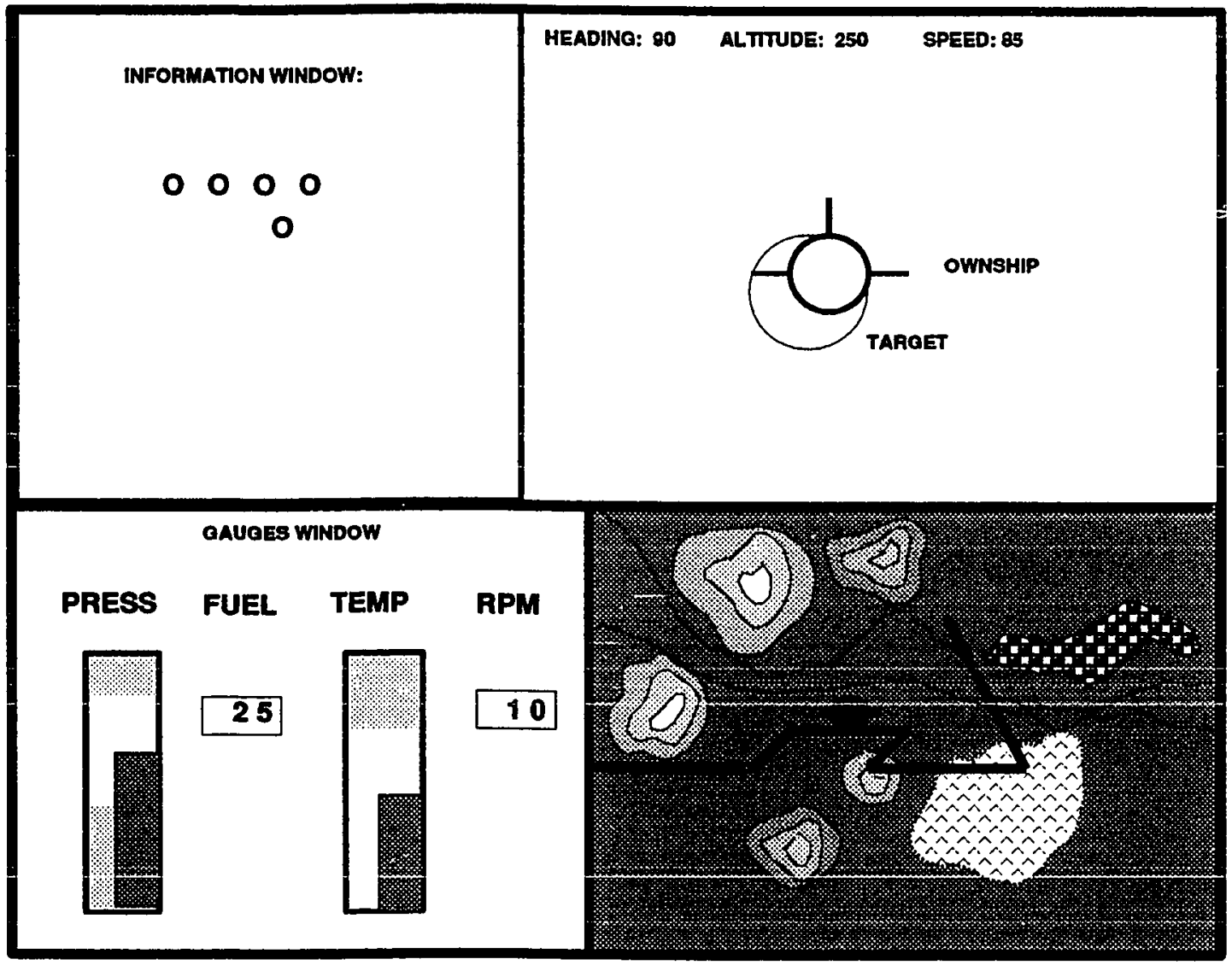

Figure 5. Example of the monitor display. 
The upper right quadrant was used to display the movement of the target for the three-axis tracking task. The three axes were heading, altitude, and speed. The top of this quadrant presented digital readings of the subjects' present heading, altitude and speed. This was a continuous task which was representative of a flight task. The goal was to match position (heading and altitude) and size (speөd) of a target and the subjects' tracking aircraft. Ideal tracking was achieved when the aircraft and the target were the same size and in the same position on the screen.

The bottom right comer of the display was used for presentation of a background map which indicated the path of the target being tracked, geographical features, and the current location of the target aircraft. The scenario determined the path of progress (flight path) through this map. The lower left quadrant displayed two vertical bars used as gauges representing oil pressure and engine temperature, and two digital read-outs representing fuel weight and revolutions per minute (RPM). In order to lend reality to the simulation the gauges were utilized but were not related to the actual tracking task or to the digital tracking read-outs. The values of these gauges were determined by the scenario and did not affect tracking.

The upper left window was used to present alphanumeric messages on the screen. This window was also used to display the discrete tasks. The discrete tasks were divided into two categories, verbal and spatial. There were two distinct tasks in each category with two levels of difficulty for each task. The verbal and spatial tasks were simllar, but required elther verbal or spatial central processing (Wlckens, 1980). The tasks were a four-choice reaction time (CRT) task and a memory recall task (MRT) with two levels of difficulty (easy and hard) for each task. 


\section{Discrete Tasks}

noupreliminary study was conducted in order to determine which items were to be used for the discrete tasks. Specific items were presented to ten subjects and reaction time data were collected. Within each level of difficulty (easy and hard) an attempt was made to equalize the tasks requiring spatial and verbal resources (i.e., the verbal task items had similar reaction times to the spatial task items for each level of difficulty). The preliminary study did not address error rate as it was assumed that with training the error rate would be minimal.

The easy spatial four-choice reaction time task was presented by displaying four O's (letter O) across one line of the alphanumeric quadrant with another $O$ used as a pointer under one of the four O's (see Figure 6a). The subjects' task was to choose one of the four buttons which was in the same position as the $O$ pointer. The difficulty of this task was manipulated by adding one or two arrows $(>>$ ) pointing to the left or right below the $O$ pointer (see Figure $6 b$ ). The subjects were required to choose the button either one or two O's to the right or the left of the pointer, depending on the number of arrows below the pointer.

The easy verbal version of the CRT task presented a single digit (one through four), corresponding to the buttons one through four (see Figure $6 \mathrm{c}$ ). When the single digit appeared, the subjects' were required to press the corresponding button. For example, if the digit 4 were displayed, the subjects were expected to respond by pressing the button labeled 4 . The difficulty of this task was manipulated by placing an equation on the screen which, when solved, equaled $1,2,3$, or 4 . Subjects were required to solve the equation and press the corresponding button number (see Figure 6d). 


\section{ALL ANSWERS IN THE EXAMPLES \\ ARE BUTTON \#2}

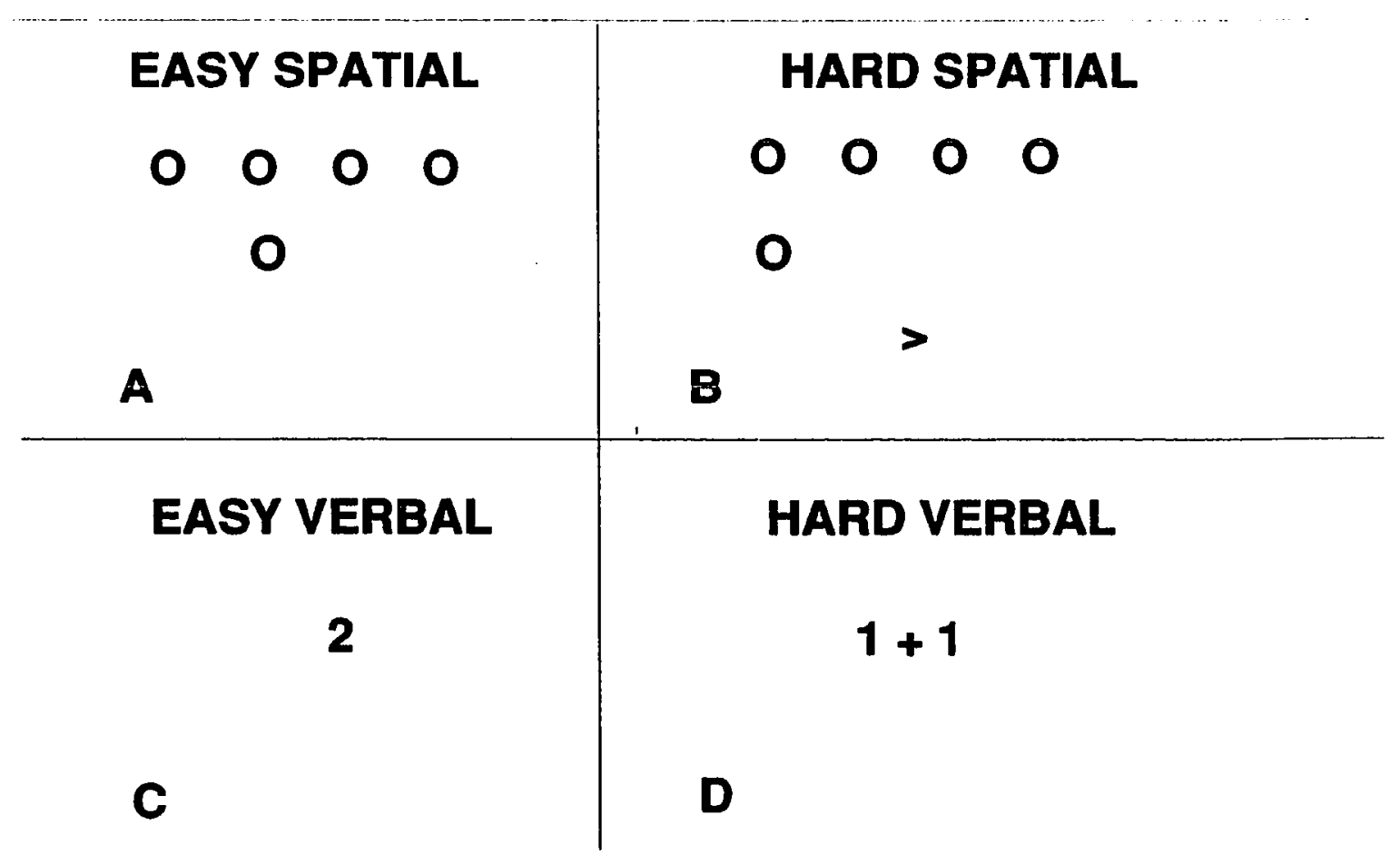

Figure 6. Examples of the CRT task. 
The second task was a memory recall task utilizing a reference presented for four seconds with a probe presented two seconds later. The subjects were required to respond "yes" if the reference and probe were the same and "no" if the reference and probe were different.

The easy spatial task consisted of a stimulus with two rows of non-alphanumeric characters. The difficulty level was increased by adding one row of characters and creating more subtle differences (only one character was different) between the stimulus and the probe. (See Appendix B for a list of all stimulus and probe pairs in both difficulty conditions.)

The stimuli for the easy verbal version of the memory recall task contained two alphanumeric characters with the first being a letter and the second being a number (e.g., P4). Only one item in the pair of characters changed when presented as a probe. The difficulty level was manipulated by increasing the number of alphanumeric characters to seven and changing two items. The first two were letters, the next three viere numbers and the last two were letters (e.g., PQ406RS). (See Appendix C for pairing of both levels of difficulty.)

\section{Design}

Three factors, attentional resource demands (spatial and verbal), difticulty level of the discrete task (easy and hard), and presentation of tracking (no tracking, low noise, and high noise) were fully crossed in a $2 \times 2 \times 3$ within-subjects factorial design.

The first factor was attentional resource demands with two levels (spatial and verbal) of the secondary discrete task. There were two distinct tasks for each level of the attentional resource demands independent variable. The two tasks were of different types. There was a choice reaction task (CRT) and a memory recognition task (MRT). Both of these tasks were presented for the spatial and verbal resource condition and at both levels of difficulty. Each set of tasks, 
either verbal or spatial, was presented during separate trials of the flight task and with no flight task.

The second factor, difficulty of the secondary discrete task, was manipulated by presenting the subjects with two levels, an easy and hard condition, of difficulty for each discrete task. Each difficuity level, easy and hard, was presented during separate trials of the flight task and with no flight task.

The third factor was the presentation of the tracking task. This task was presented with a low noise level, a high noise level, or tracking was not presented at all. The noise level refers to the forcing function of the tracking task. Equivalent amounts of noise were applied to heading and altitude. No noise was applied to speed. The two noise programs, one for heading and one for altitude, contained a series of floating point numbers in standard MicroSoft format. These numbers were properly scaled for the respective axes of the display. In order to avoid jumps on the screen and have believable noise, the numbers used in the noise cycle were sufficiently close for a smooth effect. The noise level was referred to as low and high wind for the subjects' clarification.

A control condition was provided for baseline information by running subjects on the tracking task alone for each of the two levels of noise.

\section{Dependent Measures}

Reaction time and error rate of the responses were collected on the discrete tasks during training and testing. Performance (root mean square error (RMSE) for heading, altitude and speed) on the continuous tracking task was obtained every 100 milliseconds. The tracking data were then averaged over each of eight one-minute segments. Subjective measurements of overall workload ratings were collected after each trial for the entire dual task as well as for each 
of the components of the dual task. These components were speed, heading, altitude, discrete Task 1, and discrete Task 2.

\section{Procedure}

Instructions.

Subjects were given instructions concerning the overall experiment, including a general description of the tasks and the overall workload measurements. Specialized instructions pertaining to the continuous and discrete tasks were presented immediately before the start of the trial for each condition. Subjects read the instructions (see Appendix D) to themselves and any questions were answered. Verbal instructions for the experimental trials were the same as for the practice trials. Immediately preceding each trial presented without tracking the subjects were told to use their left hand only when responding to questions. This was requested for consistency since subjects responded with their left hand while tracking. The practice trials did not have a discrete task only condition. Additional instructions were provided for the component ratings for the experimental trials since these were not utilized during the practice trials.

\section{Script Format.}

The flight task was divided into eight segments. Each segment lasted one minute and represented a different axis change and movement. As an example, segment one contained a speed change only, and segment three contained a speed and heading change (see Appendix $E$ for all segment definitions). There were three replications of the CRT and MRT tasks within a segment. The CRT and MRT lasks were presented at seven-second intervals. Each message remained on the screen for three seconds. The window remained blank for four seconds before the next task was presented. The MRT was a two-step task with a reference and probe which were presented three seconds apart. The reference was displayed for three seconds; the window 
was blank for three seconds; the probe was displayed for three seconds. As with the CRT, the window remained blank for four seconds before the next task was presented.

Practice trials.

There were six practice trials which followed an initial demonstration trial. These practice trials used a more complex flight path than the demonstration flight path. The flight path was similar in format to that used for the experimental trials.

Subjects were presented with a practice demonstration of a simple tracking task with no noise and two samples of each type of the discrete task at each level of difficulty. Each task was explained and subjects demonstrated their understanding of the discrete task by responding appropriately to the questions. Subjects also had to demonstrate that they understood the operation of the heading, altitude, and speed controls. This initlal demonstration lasted approximately five minutes depending on each subject's understanding of the overall requirements of the task. Once the subjects felt comfortable with what was expected and how the controls and buttons worked, the demonstration script was restarted and the subjects ran the trial independently. The experimenter observed each subject's performance on the experimenter's monitor, which was located outside of the subject's booth, and noted and discussed with the subject any possible misunderstanding or confusion about tracking.

After the demonstration trial, the subjects were presented with performance feedback on both the tracking and the discrete tasks. Performance on the discrete tasks was displayed on the monitor and showed the percent correct for each type of task. Tracking periormance was represented by a graph for each axis of control. A straight line through the center of each graph represented perfect performance and deviations around this line indicated the amount of error in the tracking performance. The displayed range was + or $-24 \mathrm{kts}$ for speed, + or -100 feet for 
altitude, + or - $\mathbf{4 0}$ degrees for heading. The three tracking graphs were presented in one screen on the monitor. Discrete task performance was presented first and the tracking performance was displayed second.

The feedback was discussed with the subjects. The experimenter queried the subjects on any incorrect answers in the discrete tasks. This was to ascertain that errors were not made due to a misunderstanding of the task. The main focus of the practice trials was to ensure that the subjects had a full understanding of the tasks.

The practice trials became progressively more difficult. The scenario flight path was the same for all practice trials. The first practice trial consisted of tracking only with no noise and no discrete tasks. The second trial added all of the discrete tasks at both levels of difficulty and no noise on tracking. By presenting all of the discrete tasks in this trial, the experimenter had a chance to double check any subjects' understanding of the tasks.

The third and fourth trials separated the verbal and spatial discrete tasks, but included both levels of difficulty for each. Low level noise was added to the tracking task. The fifth and sixth trials added high noise to the tracking task and also separated the verbal and spatial tasks as in trials three and four. Subjects one through six received the spatial tasks first and subjects seven through twelve received the verbal tasks first for both levels of noise (low and high). Feedback on the monitor was provided as well as from the experimenter after each practice trial.

After the practice trials the subjects were asked how they felt about the practice, whether they understood the task, and if they felt competent to continue. All subjects demonstrated a consistent level of performance by the fifth and sixth trials. A consistent level of performance was achieved when subjects were able to score above $85 \%$ correct on the discrete tasks, were able to make heading, altitude, and speed changes in the correct direction, and not to make erratic 
changes during tracking. All subjects felt they were practiced enough to continue on to the experimental sessions. All subjects were given a ten to fifteen minute break after the practice trials.

Each subject participated in one session lasting approximately five and one-half hours including breaks and instructions. The practice trials required approximately one and one-half hours. The experimental trials took approximately two and one-half hours.

Experimental trials.

The same flight script was used throughout all of the experimental trials. Each of the discrete task sets were presented at two levels of difficulty (easy and hard), at two levels of attentional demands (verbal and spatial) and at three levels of tracking presentation (low noise, high noise, no tracking). Each experimental session consisted of 14 trials. Twelve of the trials were derived from the $2 \times 2 \times 3$ design. Two additional trials were run in order to determine a baseline for the tracking task (tracking alone at each of two noise levels). A script determined the flight activities of the tracking tasks and the presentation of the discrete tasks. A trial lasted $8 \mathrm{~min}$ and consisted of one script, with one category of discrete tasks (verbal or spatlal), one level of difficulty (easy or hard), and ong of the three tracking task levels (low noise, high noise, no tracking). The order of trials was randomized. The presentation order of the discrete tasks was randomized within each trial. Each subject received all experimental conditions. Subjects were allowed breaks when they needed to stretch or rest their eyes. Most subjects required about two five-minute breaks throughout the experimental trials. One subject opted to take no breaks.

After each trial, performance feedback was first presented for the discrete task and then for tracking. The feedback was in the same format as for the practice trials. After the performance 
feedback was presented, workload ratings of the five components of the complex task and the whole task were collected.

\section{Results}

Performance and overall workload measurements of the discrete tasks were collected from a complete 2 (difficulty) by 2 (resource) by 3 (task) balanced design. In order to evaluate subjects' performance on the discrete task, reaction time and percentage of errors were analyzed. Three separate four-way within-subjects analyses of variance were performed on the dependent variables of reaction time, error rate, and component workload ratings of the discrete tasks.

The performance measurements of the tracking data were collected from a completely balanced design for each of the three axes of heading, altitude, and speed. The design was a 2 (difficulty of the discrete task) by 2 (resource of the discrete task) by 2 (level of tracking noise) by 8 (segment, changes in axes while tracking) within-subjects analysis of variance. In order to evaluate the performance of each of the tracking axes, the root mean squared error (RMSE) was analyzed. Three separate four-way within-subjects analyses of variance were performed on the dependent variables of heading RMSE, altitude RMSE, and speed RMSE.

In order to compare the component workload (CWL) ratings of the tracking axes, the three axes were treated as a task-level component (heading, altitude, and speed) independent variable. The component workload ratings were analyzed in the dual tracking condition in a separate four-way (2 [difficulty] by 2 [resource] by 2 [noise] by 3 [axis]) within-subjects analysis of variance. Tracking was presented alone: once in the low noise condition and once in the high noise condition. These two trials served as control conditions. In order to compare tracking performed alone and tracking paired with the discrete tasks, three additional separate three-way (2 [single 
and dual tracking] by 2 [noise level] by 8 [segment]) analyses of variance were performed on the dependent variables of RMSE on each axis of heading, altitude, and speed.

To compare component workload ratings of the three tracking axes when tracking alone and with tracking paired with the discrete tasks, a separate three-way (2 [single and dual tracking] by 2 [noise level] by 3 [axis]) within-subjects analysis of variance was performed.

Overall workload (OWL) ratings were analyzed in a separate three-way (2 [difficulty] by 2 [resource] by 3 [task presentation]) within-subjects analysis of variance. A second analysis of variance was performed on the OWL ratings to compare tracking alone and tracking paired with the discrete tasks.

Post-hoc Tukey comparison of means tests were utilized when indicated. The subjects factor was treated as a random variable.

\section{Discrete Task Analysis.}

Three four-way repeated measures analyses of variance for each of the discrete task dependent variables of reaction time, percentage of error, and component workload data were performed. The four independent variables were: dfficulty (easy and hard), tracking presentation (no tracking, low noise, and high noise), resource (spatial and verbal) and task type (CRT and MRT).

\section{Reaction time.}

There was a statistically significant main effect of difficulty $E(1,11)=90.81, p<.001$. The mean for the easy discrete tasks was $1242 \mathrm{~ms}$ and the mean for the hard discrete tasks was 1532 ms. Subjects' reaction times were longer for hard tasks as compared with easy tasks.

The main effect of tracking presentation was statistically significant $E(2,22)=7.17, \mathrm{p}<$ .005. A post-hoc Tukey comparison of means test was performed for the three reaction time 
means of no tracking, low noise tracking, and high noise tracking. The difference between reaction times to discrete tasks when paired with no tracking and low noise tracking was significant $(p<.01)$. There was no significant difference between reaction time to discrete tasks when paired with low and high noise on tracking. Subjects' reaction times increased when the discrete tasks were paired with tracking (low, $1430 \mathrm{~ms}$; high, $1428 \mathrm{~ms}$ ) as compared to no tracking (1304 ms), regardless of the noise level of the tracking task.

The main effect of task type was statistically significant $E(1,11)=29.42, R<.001$. The memory recognition task yielded consistently longer reaction times (mean $=1488 \mathrm{msec}$ ) than the choice reaction task (mean $=1286 \mathrm{msec}$ ).

There was no significant main effect found for resource $E(1,11)=1.062, R>.05$. There were no statistically significant differences in reaction times for discrete tasks which required verbal or spatial resources.

There was a statistically significant interaction for difficulty by resource $E(1,11)=12.60, R$ $<.005$. A post-hoc Tukey comparison of means test indicated that there was no difference between performance utilizing verbal and spatial resources in the easy condition but the difference between spatial and verbal processing in the hard condition was statistically significant $(p<.01)$. Reaction times in the hard condition tasks requiring verbal processing were longer than those requiring spatial processing. This relationship is shown in Figure 7.

There was a significant two-way interaction for difficulty by task $E(1,11)=35.99, R<.001$. The difficulty by task interaction is illustrated in Figure 8. The difference between easy and hard task levels for the MRT task was more pronounced than for the CRT task. No other significant two-way interactions were found. 


\section{DIFFICULTY BY RESOURCE INTERACTION: REACTION TIMES}

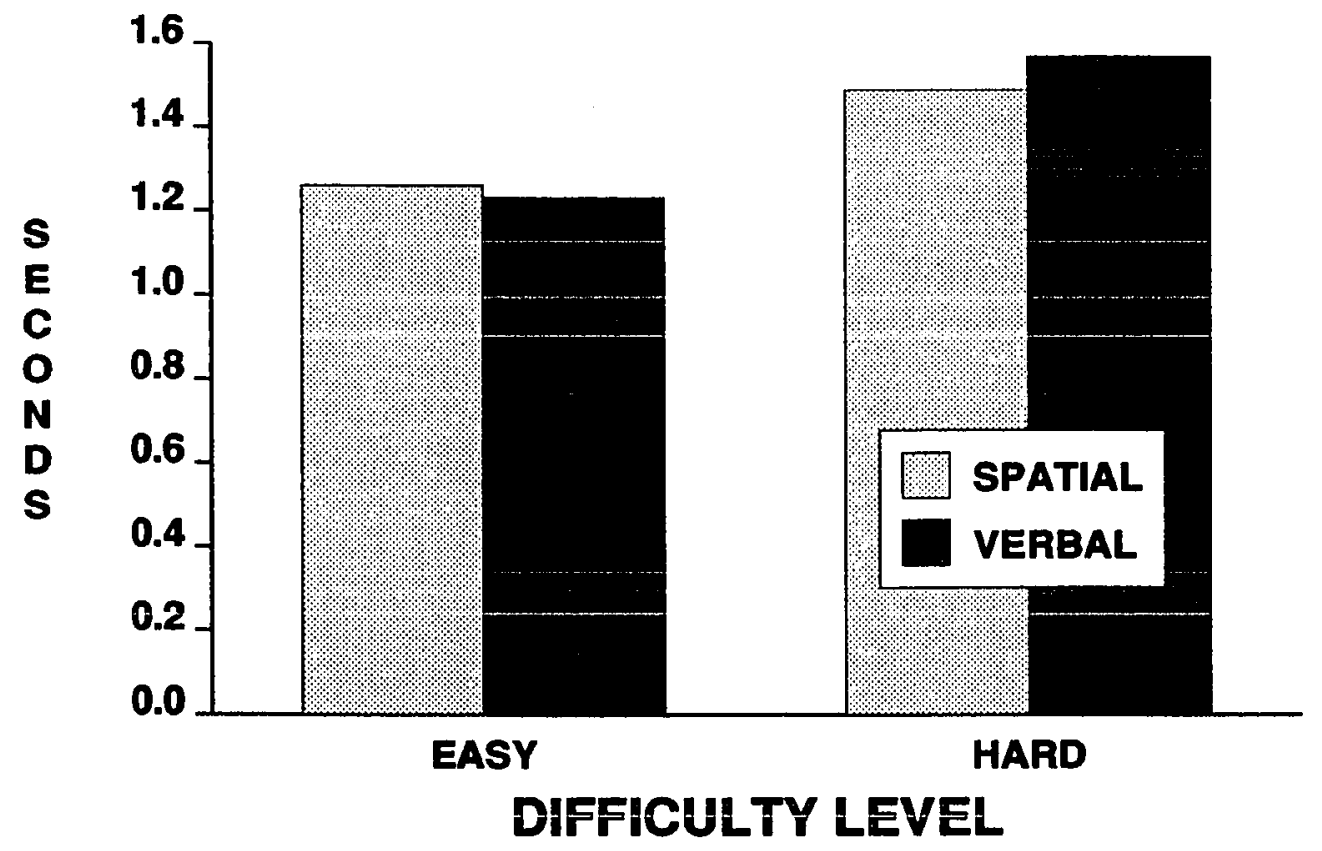

Figure 7. Reaction time as a function of difficulty and resource. 


\section{DIFFICULTY BY TASK INTERACTION: REACTION TIMES}

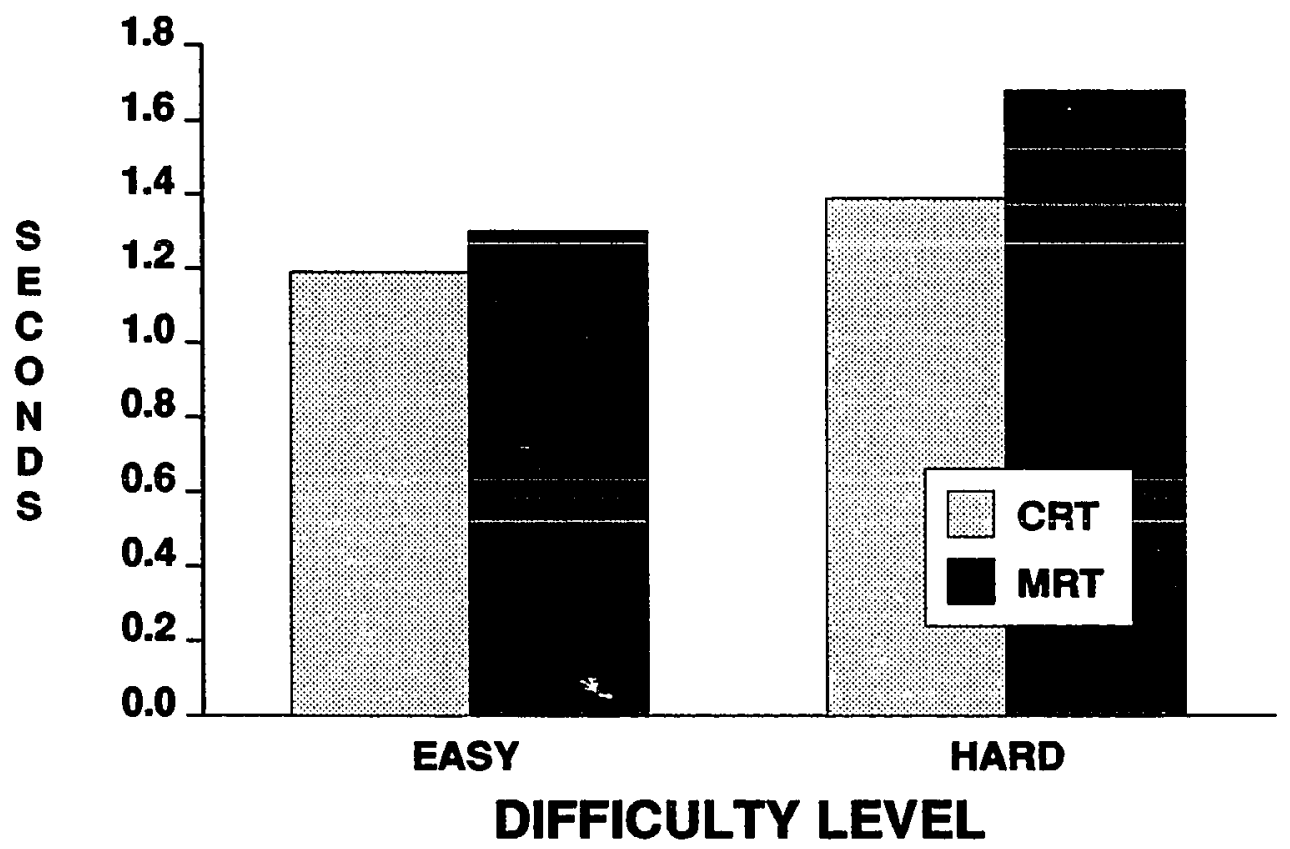

Figure 8. Reaction time as a function of difficulty and task. 
There was a significant three-way interaction for difficulty-by-resource-by-tracking presentation $E(2,22)=3.76, \mathrm{R}<.05$. A post-hoc Tukey comparison of means test was performed on the means. There was no significant difference in reaction times for verbal and spatial tasks when the discrete tasks were performed with no tracking. There was no significant difference due to difficulty of the discrete tasks for the two levels of tracking. The difference between means for low noise tracking and no tracking was significantly different $(p<.01)$ for the easy spatial, easy verbal and the hard verbal tasks. There was no significant difference at any of the levels of tracking presentation for the hard spatial task (see Figure 9). The reaction times to the hard verbal task were most affected by tracking, regardless of noise level.

Another significant thre--way interaction was found for difficulty-by-resource-by-task $E(1$, $11)=35.11, R<.01$. As shown in Figure 10, the MRT task yielded longer reaction times in the high difficulty level for the verbal resource task than for the spatial resource task. This was reversed in the low difficulty level, with longer reaction times recorded for the MRT task in the spatial resource task than for the verbal resource task. The results for the CRT task were consistent since both spatial and verbal tasks yielded higher reaction times in the hard difficulty level as compared to the easy level. Reaction times to the CRT task were similar for the verbal and spatial conditions for both levels of difficulty.

\section{Percentage of Error}

There was a significant main effect of difficulty, $E(1,11)=20.75, p<.001$. Subjects' mean percentage of errors was higher in the hard difficulty level $(6.6 \%)$ than in the easy level $(3.8 \%)$ of the discrete tasks. 


\section{DIFFICULTY BY RESOURCE BY TRACK PRES: REACTION TIMES}

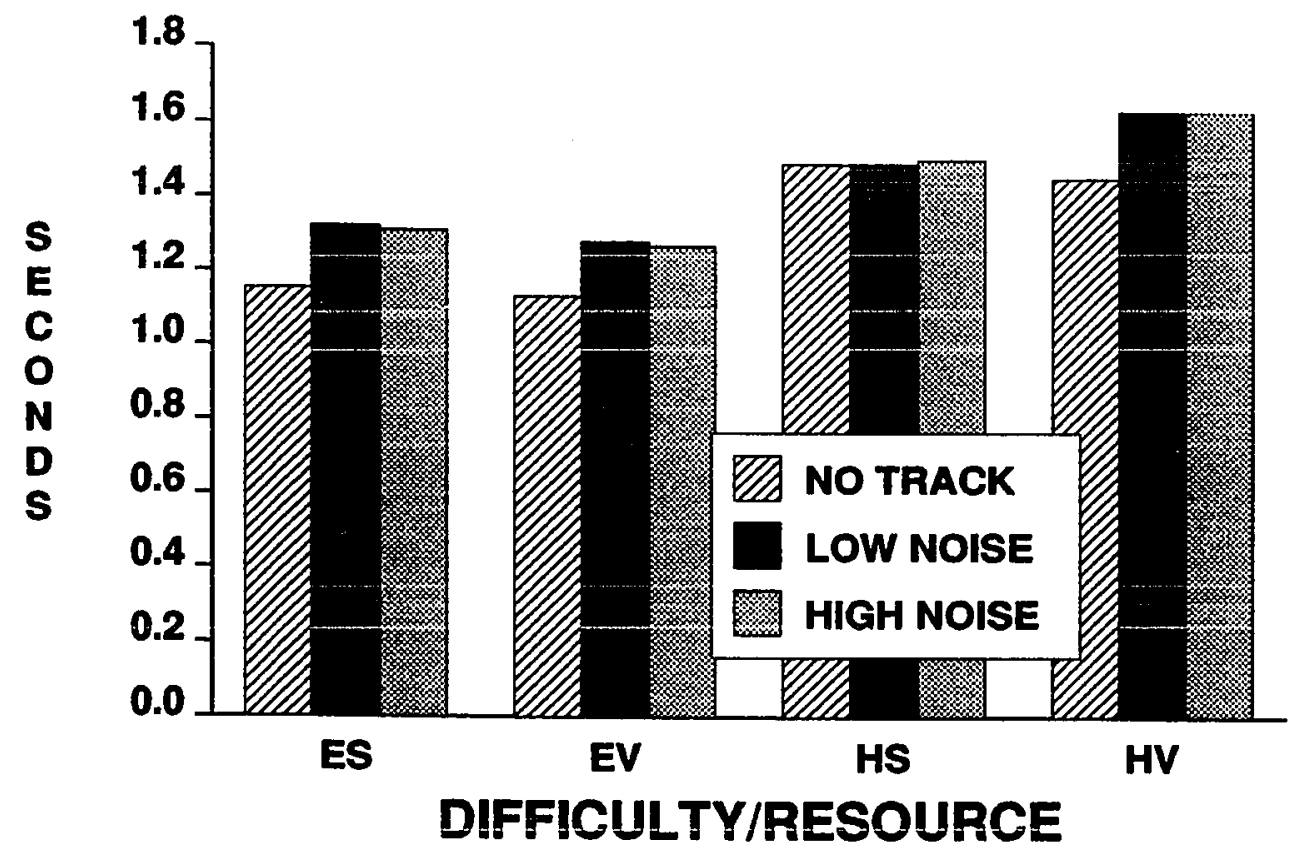

Figure 9. Reaction time as a function of difficulty, resource and tracking presentation. 


\section{DIFFICULTY BY RESOURCE BY TASK: REACTION TIMES}

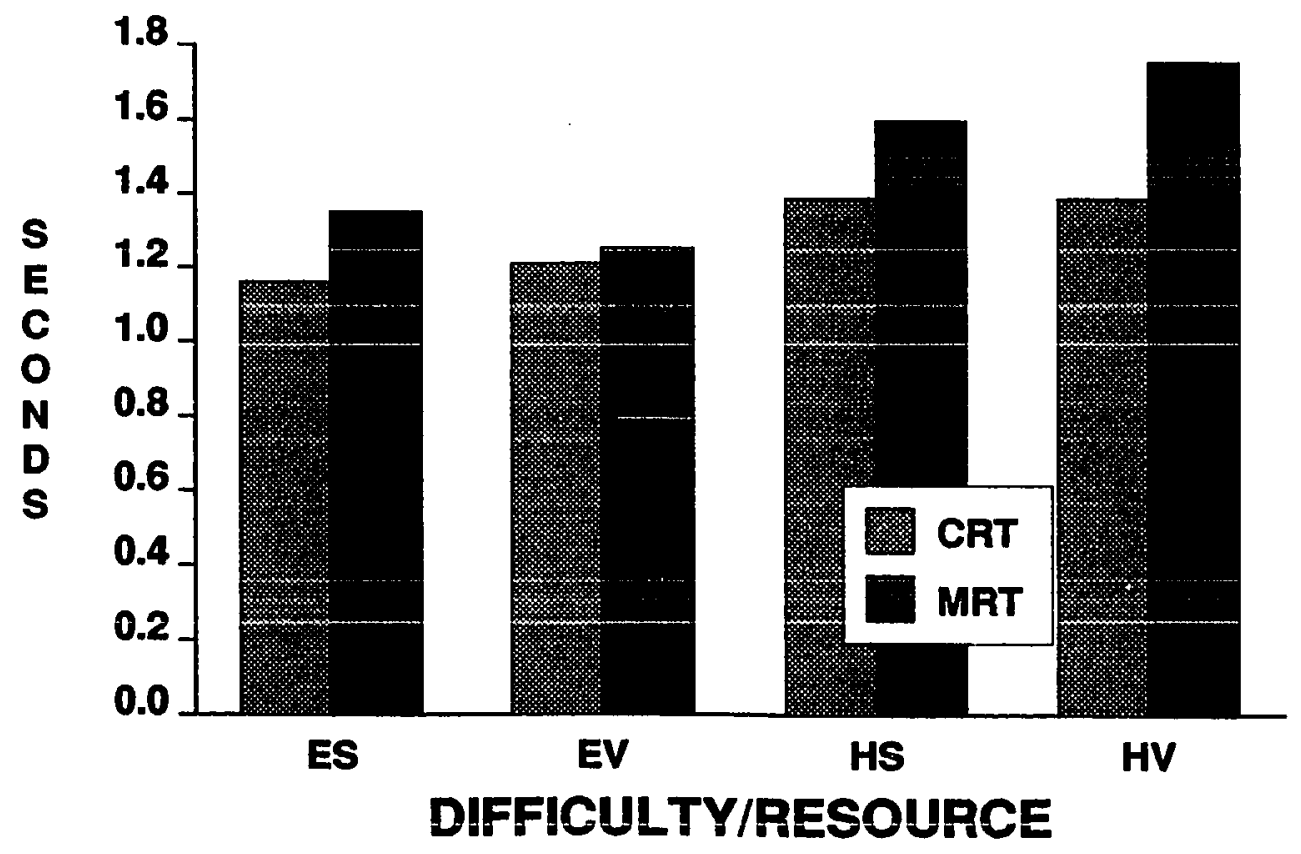

Figure 10. Reaction time as a function of difficulty, resource and task. 
There was a statistically significant main effect found for resource $E(1,11)=18.14, R<$ .001 . The spatial resource tasks had a higher percentage of error $(6.8 \%)$ than the verbal resource tasks (3.6\%).

The main effect of tracking presentation was statistically significant, $E(2,22)=4.74, p<$ .05. A Tukey post-hoc comparison of the two means for noise level indicated there was no signiffcant difference between the low and high levels of noise. Subjects' percentage of error increased when the discrete tasks were paired with tracking as compared to no tracking $(3.74 \%$ errors), regardless of the noise level of the tracking task (low, $5.6 \%$; high, $6.2 \%$ ).

The main effect of task type was significant, $E(1,11)=38.07, R<.001$. The MRT yielded a consistently higher percentage of error (9.2\%) than the CRT (1.2\%).

There was a significant two-way interaction for difficulty by task $E(1,11)=10.08, p<.01$. The difficulty-by-task interaction indicates there was a greater difference in error rate between the CRT and the MRT in the hard condition than in the easy condition. This relationship is shown in Figure 11.

There was a significant wo-way interaction for resource by task $\mathrm{E}(1,11)=23.69, \mathrm{R}<$ .001. This interaction is illustrated in Figure 12. The difference in error rate between tasks requiring spatial or verbal resources was more pronounced for the MRT than for the CRT.

There was a significant two-way interaction for tracking presentation by task $E(2,22)=$ 5.19, $\mathrm{R}<.01$. A post-hoc Tukey comparison of means test was performed between the means of error rate for the CRT and MRT. The results indicated that there was no significant difference for the CRT between means for the three levels of tracking presentation. However, there was a significant difference for the MRT between means when compared to single task and low noise tracking $(\mathrm{g}<.05)$. Although not statistically significant, the error rate was higher in the high noise 
DIFFICULTY BY TASK:

ERROR RATE

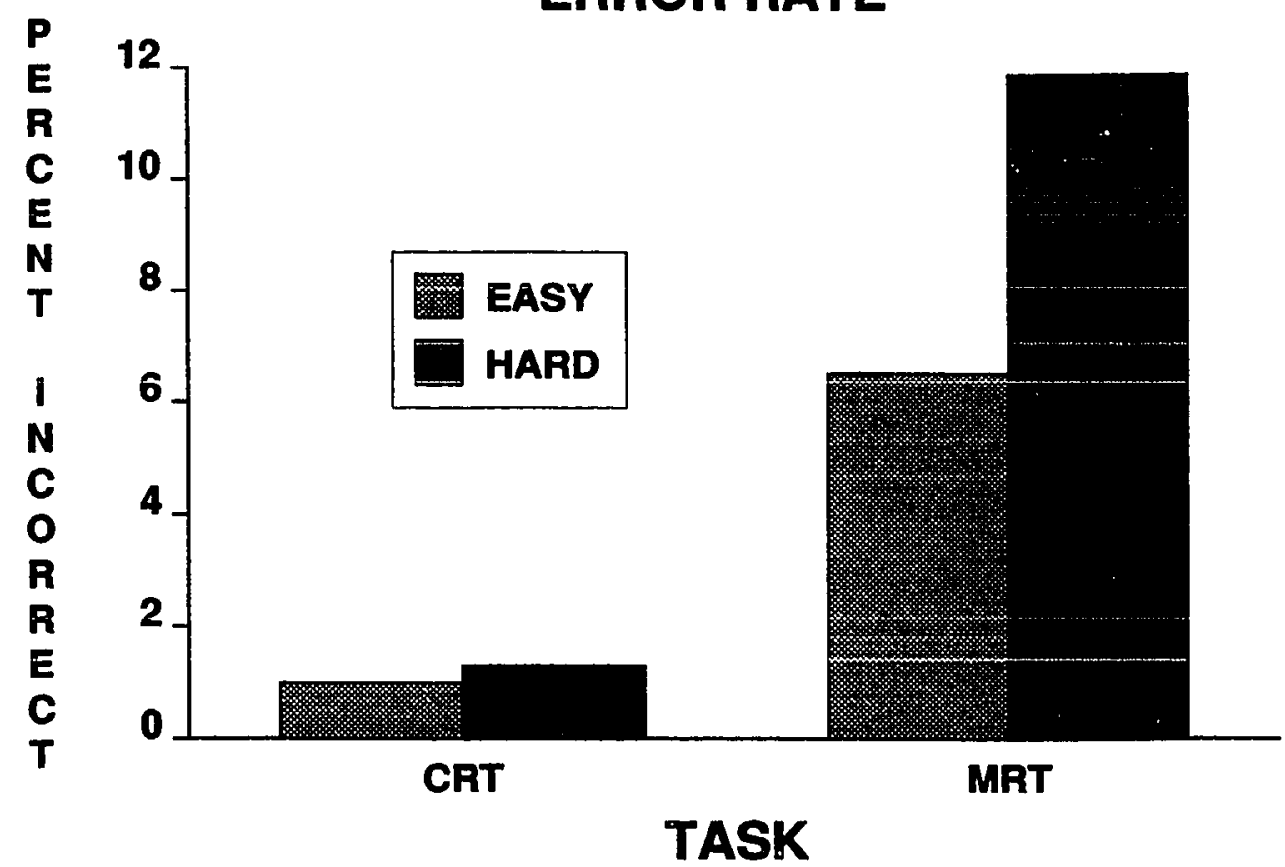

Figure 11. Error rate as a function of difficulty and task. 


\section{RESOURCE BY TASK:} ERROR RATE

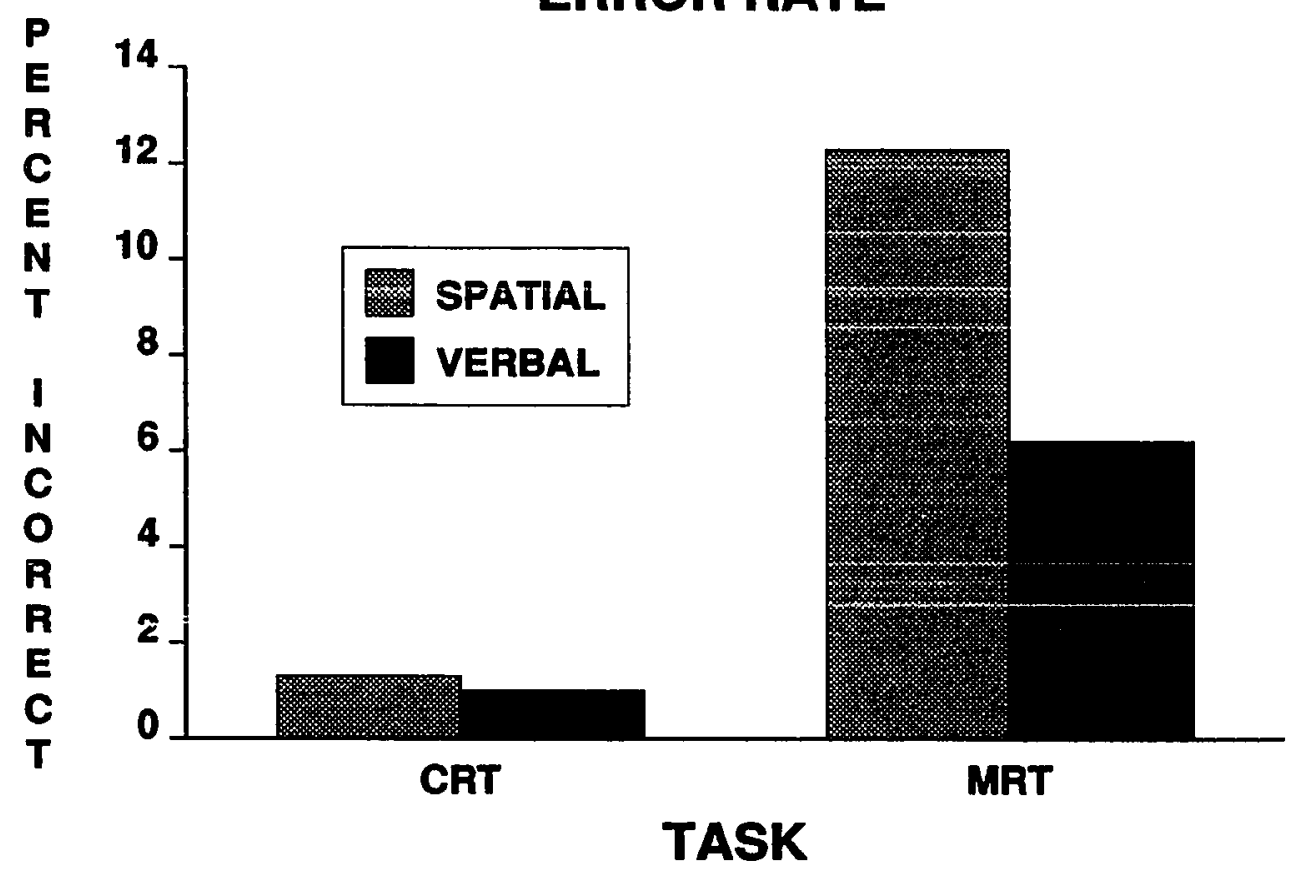

Figure 12. Error raie as a function of resource and task. 
level than in the low noise level. As shown in Figure 13, the percentage of errors for MRT was affected by the tracking presentation more than the CRT. No other statistically significant interactions were found.

Component Workoad Ratings of the Discrete Tasks

There was a significant main effect of difficulty on workload ratings, $E(1,11)=8.20, \mathrm{R}<$ .05. Subjects' component workload ratings were higher for the hard discrete tasks (35.0) as compared with lower ratings for the easy discrete tasks (28.9).

The main effect of tracking presentation was significant, $E(2,22)=4.68, p<.05$. Subjects' workload ratings increased as task demand increased. This relationship is illustrated by the means (no track, 28.6; low noise, 31.8; high noise, 35.5).

The main effect of task type was significant, $E(1,11)=18.22, p<.001$. As indicated by the means, the mean workload ratings for the memory recognition task (39.5) was higher than the mean workload ratings for the choice reaction task (24.4).

There was no significant main effect found for resource $E(1,11)=1.82, p>.05$. This Indlcates there were no differences in workload ratings for tasks which required verbal or spatial resources of the discrete tasks. There were no significant interactions.

\section{Tracking Performance Data}

Deviations from the target on heading, altitude, and speed were collected every 100 miliseconds. These raw scores were separated into one-minute segments and the average root mean square error (RMSE) was computed for each one-minute segment.

Three separate four-way ( 2 [difficulty of the discrete task] by 2 [resource of the discrete task] by 2 [level of tracking, low and high noise] by 8 [segment, changes in axes while tracking]) within-subjects analyses of variance were performed on the dependent variables of heading 


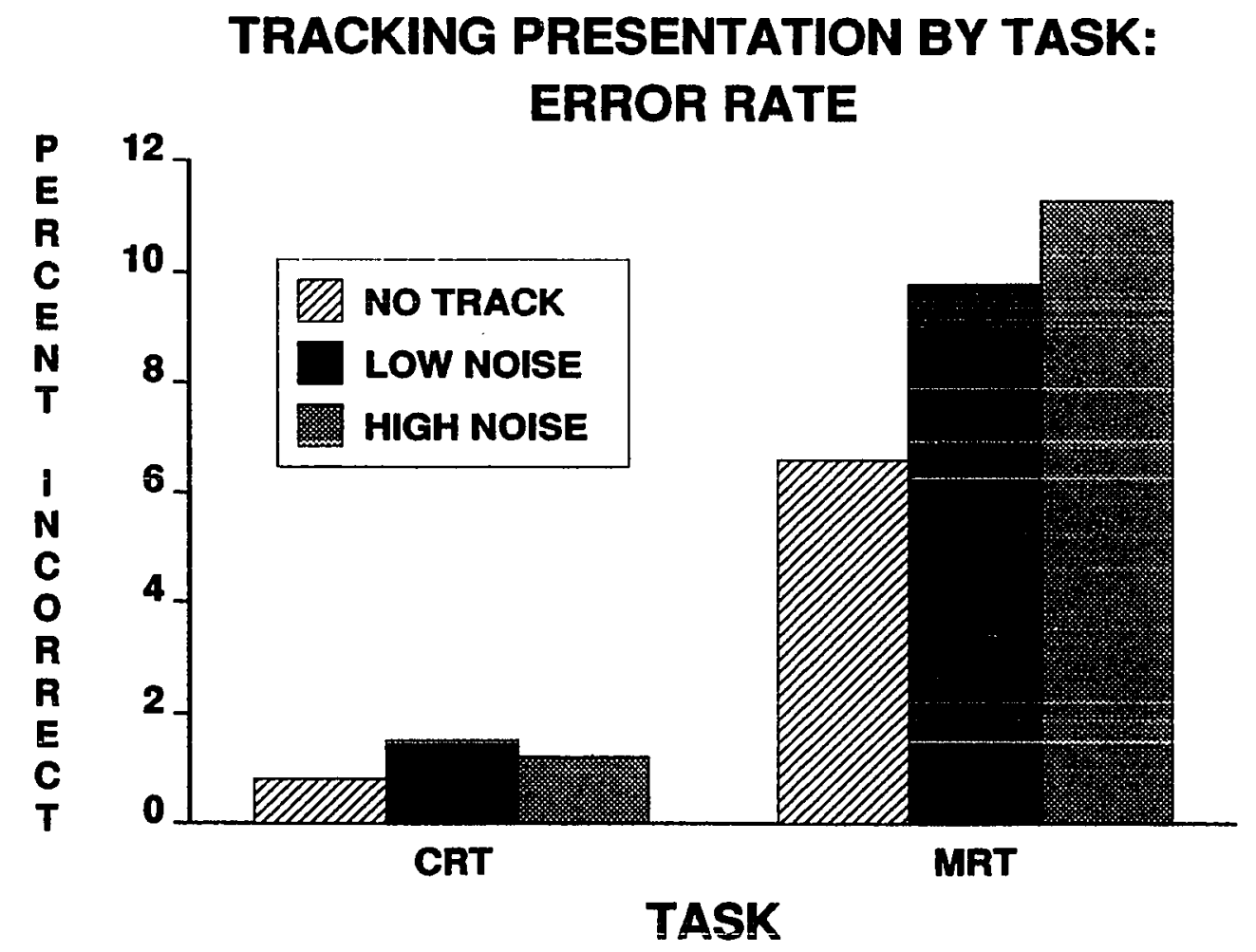

Figure 13. Error rate as a function of tracking presentation and task. 
RMSE, altitude RMSE, and speed RMSE. These analyses were performed on tracking paired with the discrete tasks.

Heading RMSE in Dual Task Condition.

There was a significant main effect due to noise $E(1,11)=163.63, p<.001$. Subjects' RMS error for tracking on heading was greater at the high noise level (2.9 degrees) than at the low noise level ( 1.6 degrees).

There was a significant main effect due to segment $E(7,77)=34.48, p<.001$. Figure 14 indicates that the heading RMSE was more affected during segments in which heading changes occurred than in segments in which there were no heading changes.

There was no significant main effect found for difficulty $F(1,11)=0.97, p>.05$. The subjects' ability to track heading was not affected by the two levels of difficulty of the discrete tasks. There also was no significant main effect found for resource $E(1,11)=0.98, p>.05$. The subjects' ability to track heading was not affected by the two levels (spatial and verbal) of resource of the discrete tasks.

There was a significant two-way interaction for noise by segment $E(7,77)=2.20, p<.05$. The subjects' ability to track heading when two or more axes were changing was more affected by the high level of noise. The increase in noise did not have as great an effect on tracking in segments in which only speed changed and no axis changed. This relationship is demonstrated in Figure 15.

\section{Altitude RMSE in Dual Task Condition}

There was a significant main effect due to noise $E(1,11)=47.38, R<.001$. The mean RMSE in the low noise condition ( 3.6 feet) was lower than the mean RMSE in the high noise condition ( 6.0 feet). 


\section{SEGMENT MAIN EFFECT: HEADING RMSE}

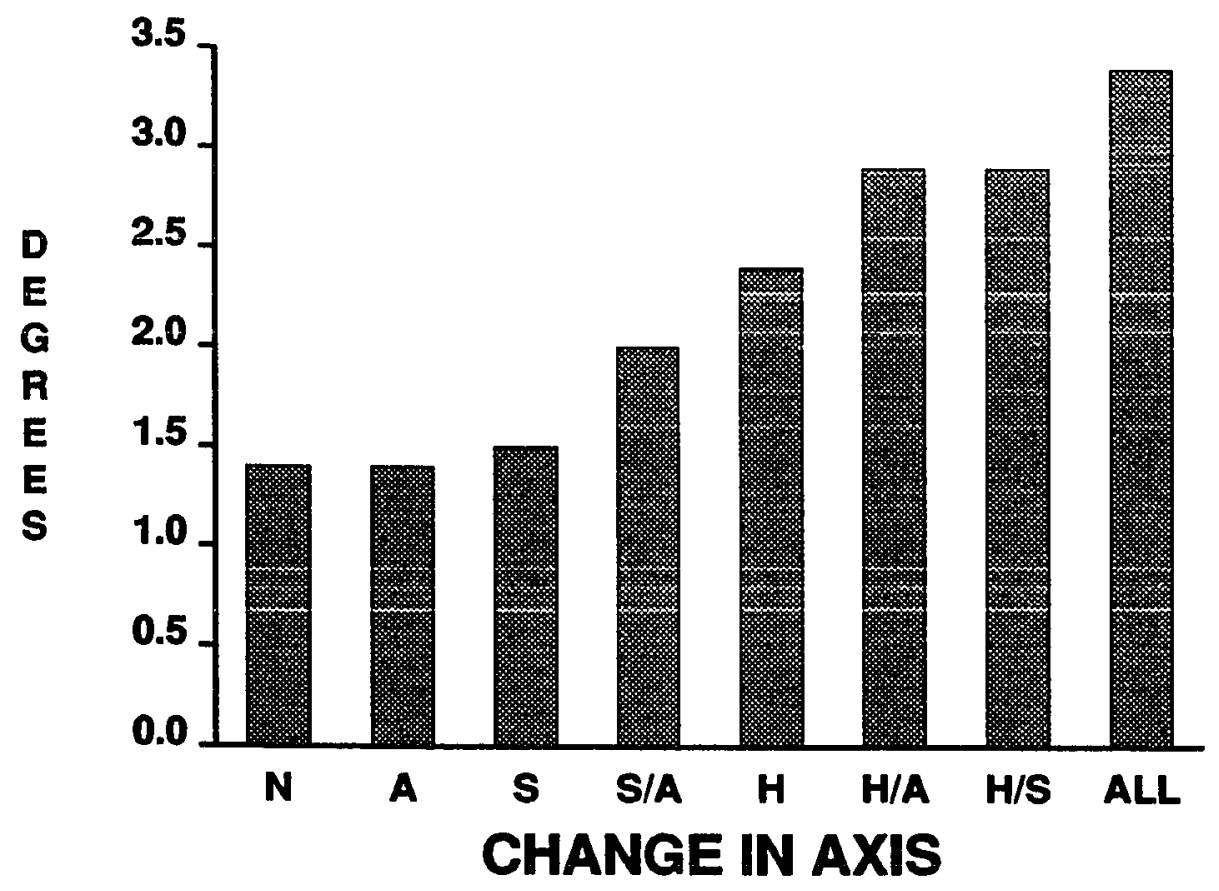

Figure 14. Heading RMSE as a function of axis changes. 


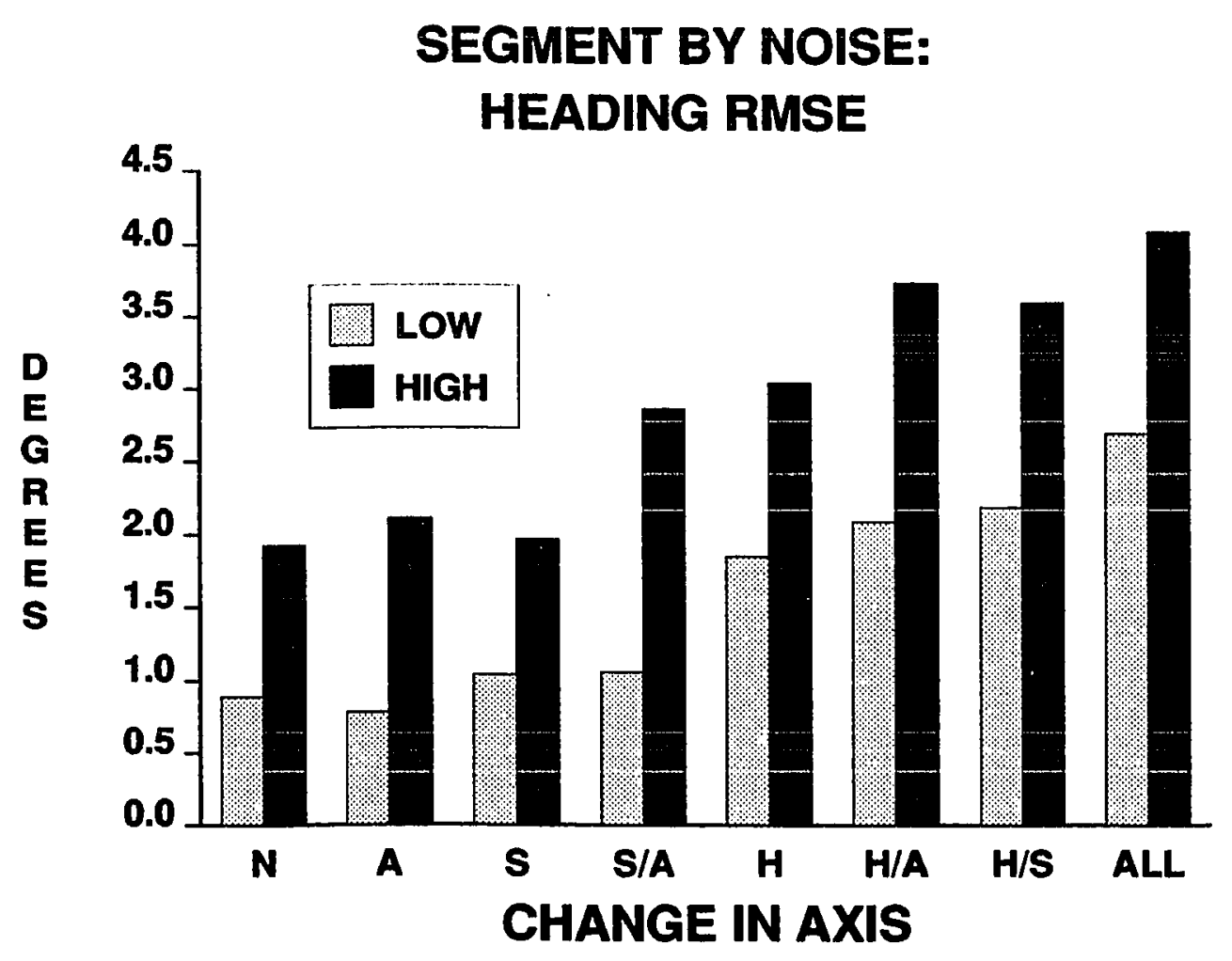

Figure 15. Heading RMSE as a function of noise and change in axis. 
There was a significant main effect due to segment $E(7,77)=11.77, p<.001]$. As Figure 16 shows, RMSE on altitude was more affected during segments in which altitude changes occurred than in segments when only heading and/or speed changes occurred.

There was no significant effect found due to difficulty $E(1,11)=1.32, R>.05$. Altitude RMSE was not affected by the levels of difficulty (easy and hard) of the discrete tasks. There also was no significant main effect found for resource $E(1,11)=3.12, p>.05$. This indicates there was no effect in altitude RMSE when paired with tasks requiring either verbal or spatial resources. There were no significant interactions.

Speed AMSE in Dual Tracking

There was a significant main effect due to noise $\mathrm{E}(1,11)=4.93, \mathrm{p}<.05$. Subjects' mean RMS error for speed was greater in the high noise level $(2.49 \mathrm{kts})$ than in the low noise level (2.29 kts).

There was a significant main effect due to segment $E(7,77)=18.64, \mathrm{R}<.001$. Figure 17 indicates that the RMSE on speed was more affected during segments in which speed changes occurred than in segments in which only heading and/or altifude changes occurred.

There was no significant main effect found for difficulty $E(1,11)=0.83, R>.05$. The subjects ability to track speed was not affected by the two levels of difficulty (easy and hard) of the discrete tasks. There was no significant main effect found for resource $E(1,11)=1.92, p>$ .05. There was no difference in speed RMSE when paired with tasks requiring either verbal or spatial resources.

There was a significant two-way interaction for difficulty by noise $E(1,11)=5.50,2<.05$. This difficulty-by-noise interaction indicates that as the difficulty of the discrete task is increased, the difference between speed RMSE at low and high noise levels was greater. A post-hoc Tukey 


\section{SEGMENT MAIN EFFECT:}

ALTITUDE RMSE

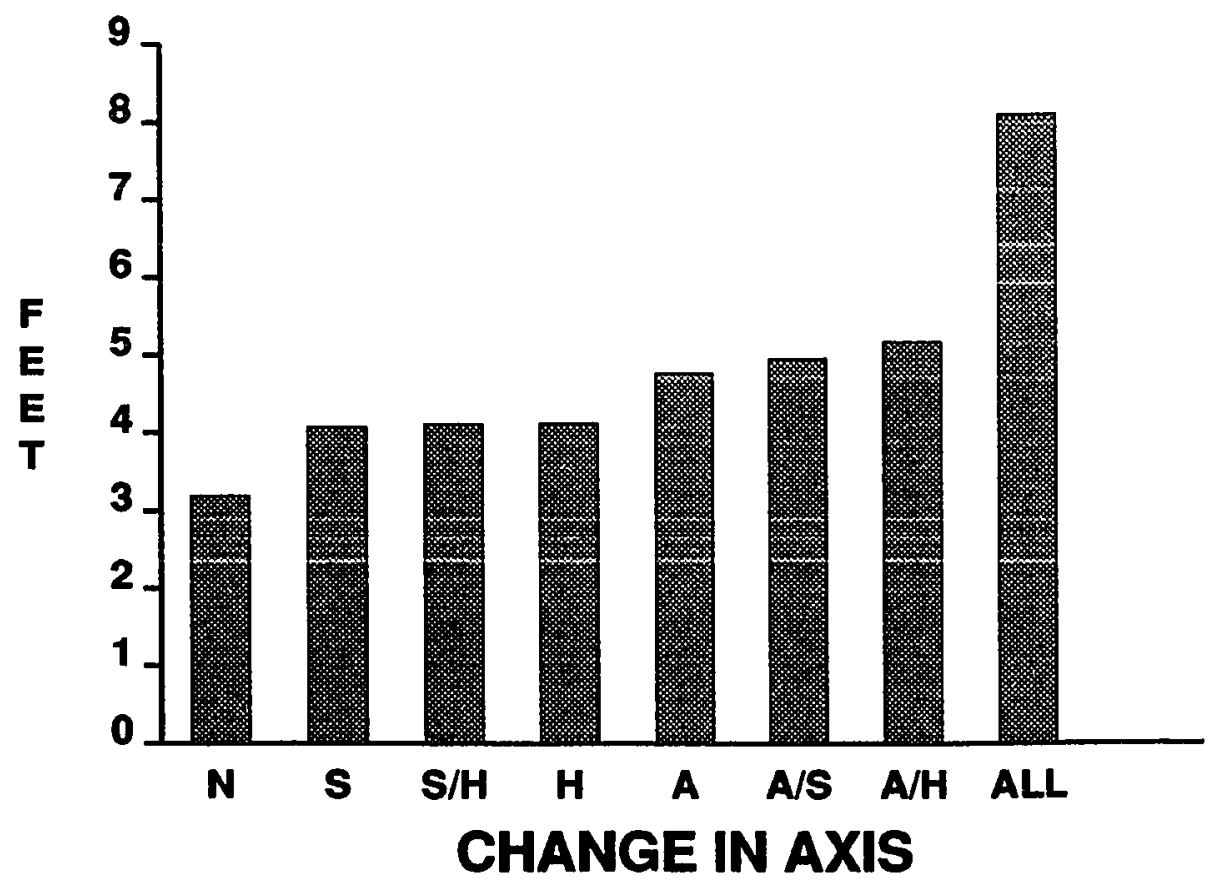

Figure 16. Altitude RMSE as a function of axis change. 


\section{SEGMENT MAIN EFFECT: SPEED RMSE}

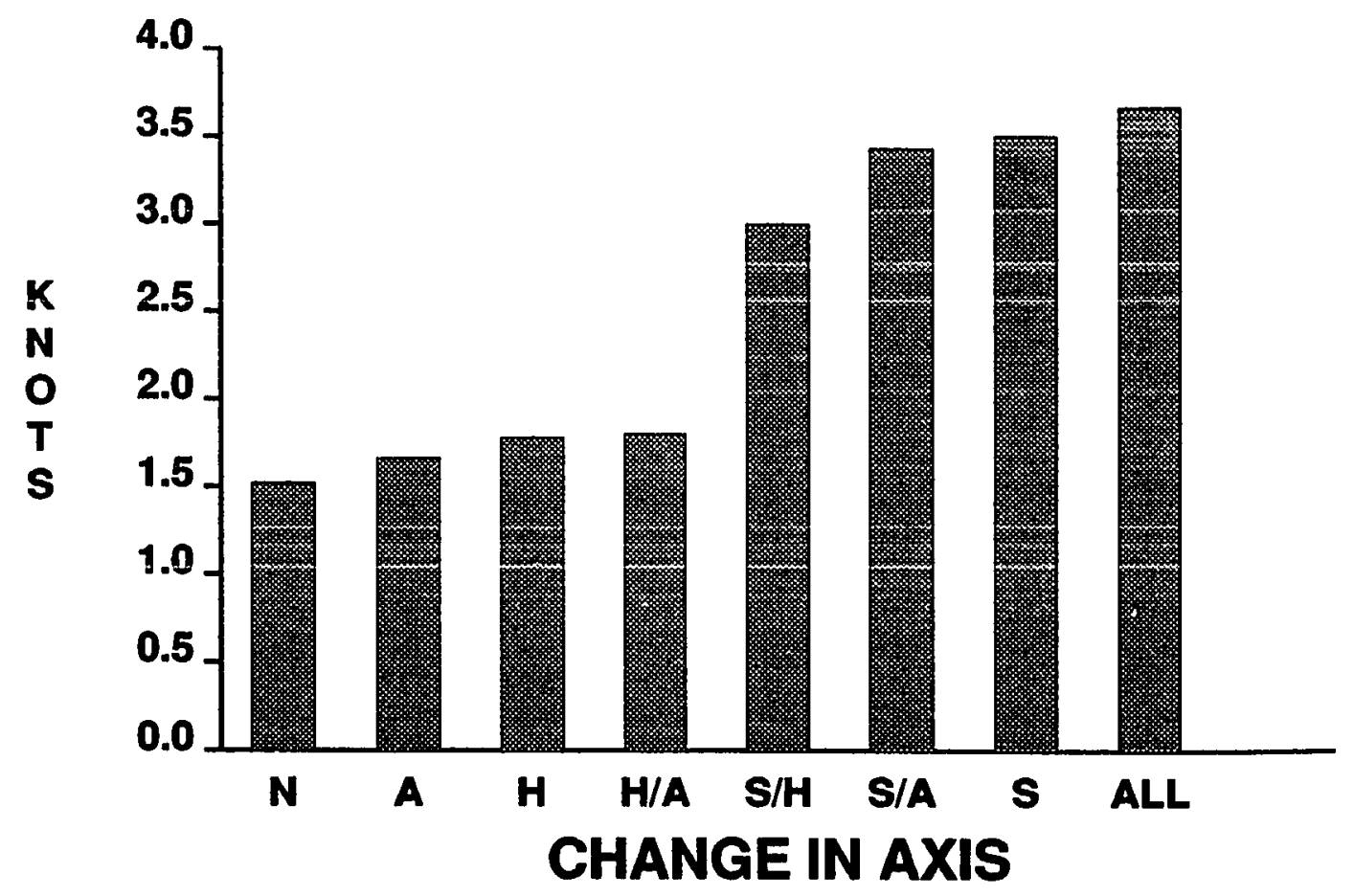

Figure 17. Speed RMSE as a function of axis change. 
comparison of means demonstrated that there was no significant difference between speed RMSE at the two noise levels in the easy condition of the discrete task. There was a significant difference between RMSE at the two noise levels for the hard condition $(R<.05)$. This relationship is demonstrated in Figure 18. There were no other interactions.

\section{Component Workload Ratings of the Tracking Axes}

A four-way ( 2 [difficulty] by 2 [resource] by 2 [noise level] by 3 [axes]) within-subjects analysis of variance was performed on the dependent variable of component workload ratings for the three axes.

There was a significant main effect due to noise $E(1,11)=14.01, R<.005$. As shown by the means, subjects' component workload ratings were higher for the high noise level (46.5) as compared with lower ratings for the low noise level (33.8) condition.

There was no statistically significant main effect for difficulty $E(1,11)=1.83, R>.05$, or for resource $E(1,11)=0.57, \mathrm{p}>.05$. Subjects' workload ratings were not affected by the difficulty or the resource of the discrete tasks.

There was no statistically significant main effect for axis $E(2,22)=0.23, p>.05$. As demonstrated by the means (heading, 40.8 ; altitude, 40.7; speed, 38.9) there was no difference in the workload ratings on any of the three axes. There were no significant interactions.

\section{Single versus Dual Tracking Performance}

It was not possible to analyze single performance measurements of tracking in the balanced design including difficulty and resource of the discrete tasks. In order to assess the effects of single versus dual tracking, three three- way $(2 \times 2)$ analysis of variance were performed to compare single versus dual task tracking performance and noise levels on the 


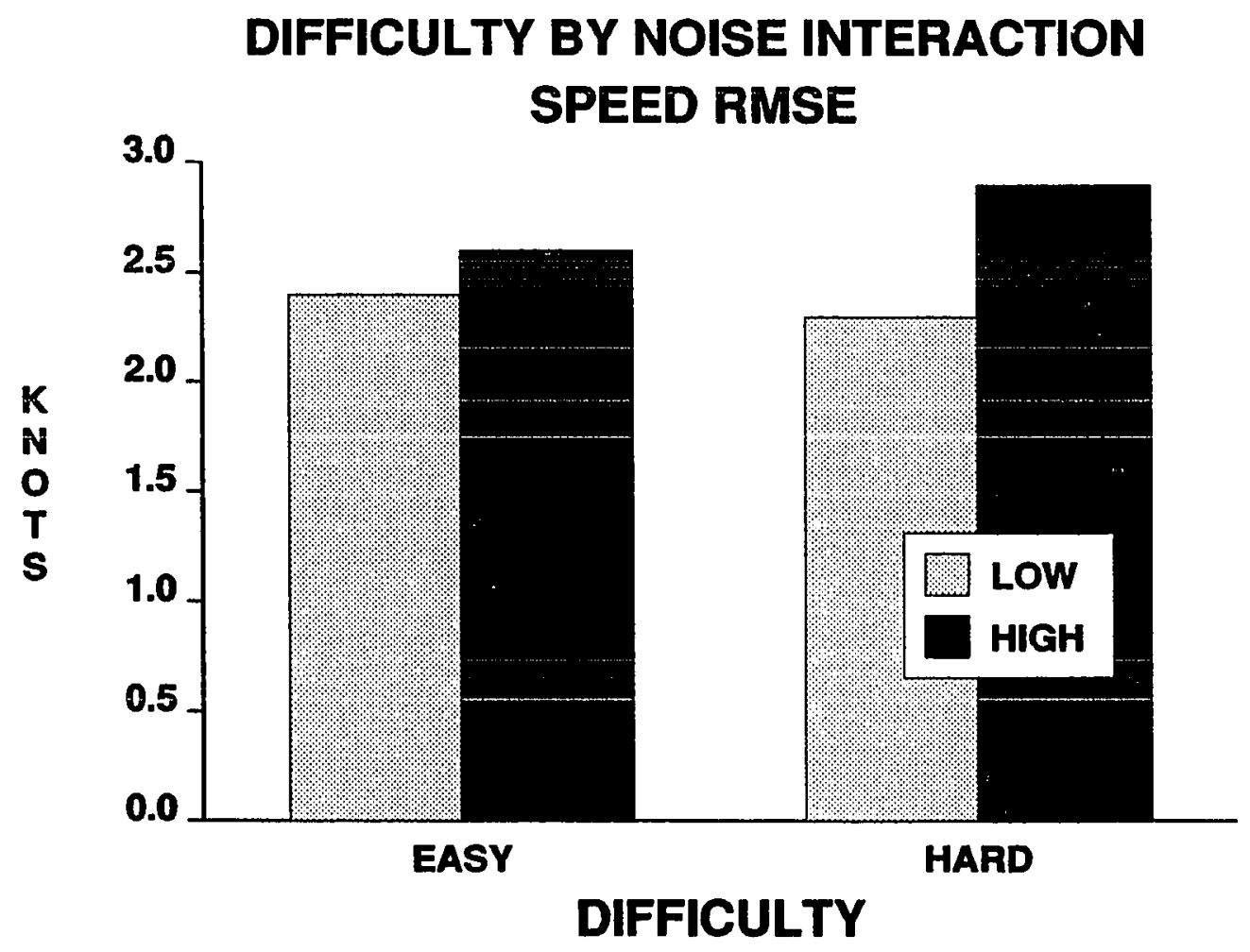

Figure 18. Speed RMSE as a function of discrete task difficulty and tracking difficulty. 
dependent variables of RMSE on heading, altitude, and speed. Single task performance refers to tracking with no discrete tasks.

Heading RMSE

There was a statistically significant main effect due to single versus dual task presentation $\mathrm{E}$ $(1,11)=14.46, R<.005$. Subjects' RMSE on heading was greater when tracking was presented with the discrete task (2.2 degrees) than when tracking was presented alone (1.9 degrees).

There was a significant main effect due to noise level $E(1,11)=262.26, p<.001$. As demonstrated by the means, tracking with low noise yielded a smaller RMSE (1.4 degrees) than tracking with high noise (2.7 degrees). There was no statistically significant interaction.

\section{Altitude RMSE}

There was a significant main effect due to single versus dual task presentation $E(1,11)=$ $9.73, p<.01$. Subjects' RMSE on altitude was greater when presented with the discrete task $(4.8$ feet) than when tracking was presented alone (4.1 feet).

There was a significant main effect for noise $E(1,11)=48.53, p<.001$. The altitude RMSE was lower in the low noise (3.3) level than in the high noise (5.6) level. There was no statistically significant interaction.

\section{Spe日d RMSE}

There was a significant main effect due to single versus dual task presentation $E(1,11)=$ 10.82, $\mathrm{R}<.01$. As the means indicate, subjects' RMSE for tracking speed was greater when presented with the discrete task (2.56 kts) than when tracking was presented alone (2.21 kts). There was no statistically significant main effect for noise $E(1,11)=1.25, R>.05$. 


\section{Component Workload Ratings of Tracking}

There was a significant main effect of workload ratings on tracking for the two lev'els of noise $E(1,11)=27.73, R<.001$. Subjects' workload was rated higher for the high noise condition (43.5) than for the low noise condition (33.3) .

There were no statistically significant main effect for single versus dual tracking $E(1,11)=$ $4.12, p>.067$, although the $E$ value approached significance with single tracking ratings (36.2) being lower than dual (40.1) task ratings. There was no statistically significant main effect due to axis $E(2,22)=0.468, p>.05$. The workload ratings were similar for heading, altitude, and speed.

\section{Overall Workload Ratings for the Complex Task}

A three-way repeated measures analysis of variance for the overall workload (OWL) ratings was performed for ratings collected when the discrete tasks were presented with tracking. The three independent variables were: difficulty (easy and hard), resource (verbal and spatial) of the discrete tasks, and tracking presentation (no tracking, low noise, and high noise).

\section{Discrete Task Condition.}

The main effect of tracking presentation was statistically significant $F(2,22)=9.91, p<$ .001. Subjects' overall workload increased when the discrete tasks were paired Wiitii iracking and as noise level increased (no tracking; 31.9, low noise; 43.3, high nolse; 50.2).

There was no significant main effect found for difficulty $E(1,11)=2.01, R>.05$, or resource $E(1,11)=1.58, R>.05$. Subjects' workload ratings were not affected by the difficulty or the resource of the discrete tasks.

There was a significant two-way interaction found for difficulty-by-tracking-presentation $F$ $(2,22)=4.23, R<.05$. A post-hoc Tukey test yielded a significant difference $(R<.01)$ between 
easy and hard levels of the discrete task for the no tracking condition. There was no significant difference between easy and hard levels of the discrete task at the low or high noise level of tracking. This relationship is illustrated in Figure 19.

Single versus Dual OWL

A two-way ( 2 [single vs. dual] by 2 [noise]) repeated measures analysis of variance for overall workload was performed to compare ratings collected in the single tracking task condition with ratings collected when tracking was paired with the discrete tasks.

There was a s:atistically significant main effect due to single versus dual task presentation $E(1,11)=15.21, p<.005$. As demonstrated by the means (single, 32.2; dual, 37.7), subjects' workload ratings for tracking increased when the tracking was paired with the discrete tasks.

There was a significant main effect on workload ratings for noise on tracking $E(1,11)=$ 8.37, $\mathrm{p}<.05$. Workload was rated higher for the high noise (37.5) condition than for the low noise (32.2) condition. There were no statistically significant interactions.

\section{Discussion}

The purpose of this study was to examine dual task additivity in terms of parformance and workload as related to several different information processing models. In order to test the various information theories, the resource model was selected to serve as the basis for the design of this experiment. Two specific hypotheses were investigated. It was hypothesized that, if the resource model is true, as difficulty increases in either the continuous or the discrete task, performance and workload will be more greally affected when the tracking task is paired with the spatial task than when it is paired with the verbal task. Performance on either the continuous or discrete task will decrement more when the discrete task involves spatial processing as a result of competition for the same resources. The workload ratings will be higher when the two tasks 


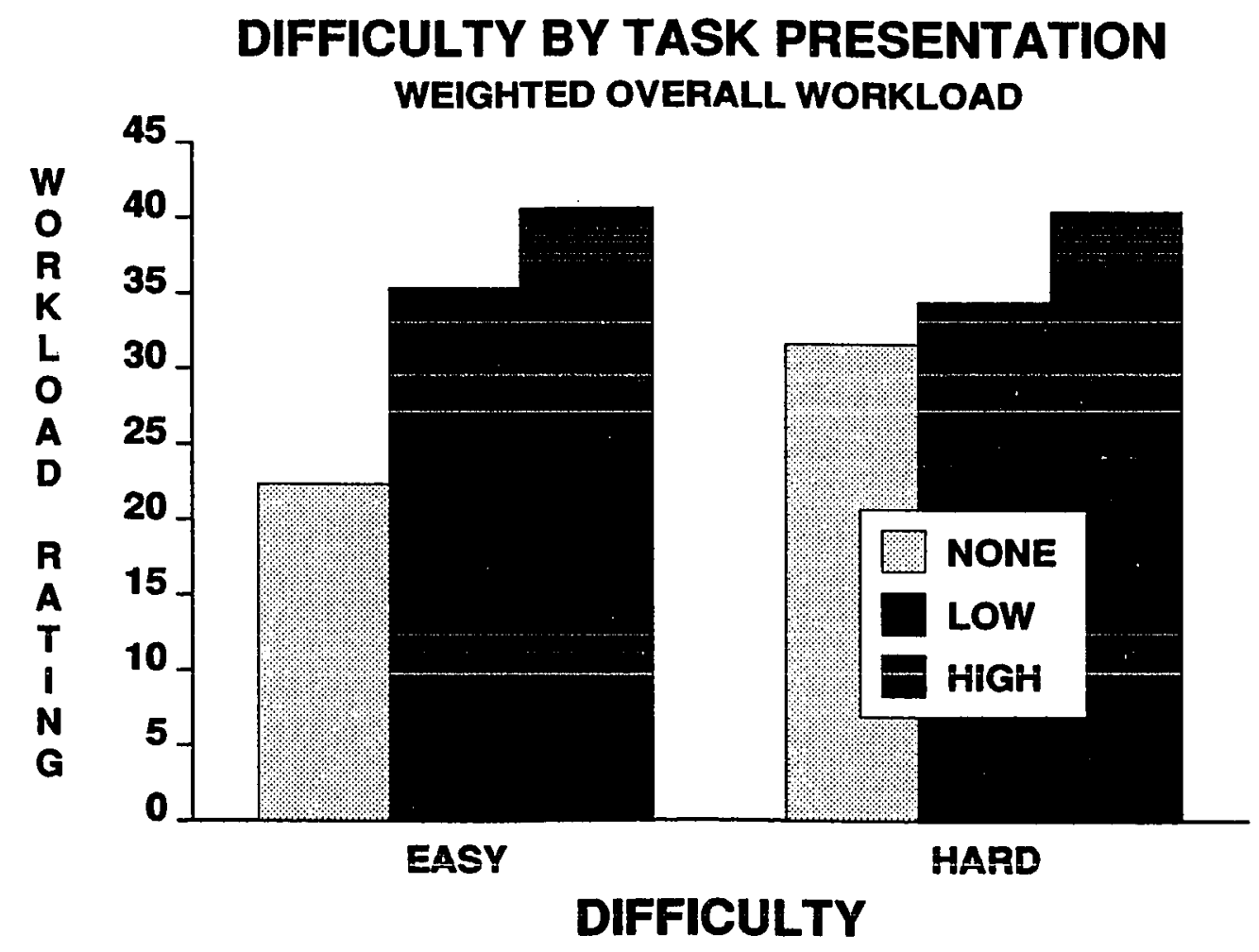

Figure 19. Workload as a function of the discrete task difficulty and tracking presentation. 
performed together require spatial resources. The other hypothesis tested was that the workload ratings of the components of the dual task would be consistent with the ratings given in the single component task condition.

The discussion of the results will be structured by examining the dependent variables: in terms of performance (reaction time and error rate) on the discrete tasks, on the tracking task (heading, altitude and speed RMSE), and on the workload component ratings and overall workload ratings. The independent variables are discussed within the section of each dependent variable.

\section{Performance on the Discrete and Tracking Task}

The statistically significant main effect of difficulty of the discrete tasks on reaction time and Giror iate data in the present experiment validated this manipulation. The easy level had significantly faster reaction times and fewer errors than the hard level.

There was no significant effect on tracking performance due to the difficulty of the discrete task. The tracking task was presented at two levels of difficulty: low and high noise. The tracking performance for all three axes (heading, altitude, and speed) was affected by the iracking presentation difficulty manipulation (i.e., noise). This effect validated the difficulty manipulation for tracking.

A single-to-dual task performance decrement indicates that the demand for available resources has increased (Wickens, 1984b). In this experiment, in order to assess whether the demand for the available resources had indeed increased, both the discrete tasks and tracking task were subjected to a single-to-dual task manipulation, in order to determine a baseline performance in the single task condition. As expected, statistically significant differences 
between single and dual task performance were obtained for both discrete task and tracking performance.

Tracking presented in the single task condition did not fit into the $2 \times 2 \times 3$ design, therefore it was presented alone as a control condition in order to compare single-to-dual task tracking performance. There was a significant difference on tracking performance for all three axes between the single and dual task conditions.

As expected, there were significant main effects on reaction time and error rate due to tracking presentation. This manipulation compared the single-to-dual task performance on the discrete tasks. It also compared the two levels of difficulty of tracking on the discrete task performance. Generally, reaction time was shorter and the error rate lower for the discrete task when it was performed alone than when it was paired with tracking. However, the reaction times and error rates of the discrete tasks were not affected by the two levels of tracking difficulty. Although the discrete task performance was affected by the presence of a second task (tracking), the performance was not affected by the increased demand of that second task.

Contrary to the predictions of the multiple resource model hypothesis, tracking performance did not decline as a result of increased difficulty of the discrete tasks; nor was there a significant effect on the reaction time and error rate of the discrete task attributed to the difficulty manipulation of the tracking task. It was expected that as the difficulty level of the spatial discrete task increased, performance on the tracking task would decrement and this result was not found. Thie spatial tracking task did not differentially affect the verbal or spatial discrete task. This could indicate that subjects are willing to work harder and may increase their available resources through effort (Kahneman, 1973) or that there is no competition for the same resources, but rather a limited amount of resources in general (Kantowitz, 1988). 
Two types of discrete tasks were utilized in this experiment for greater generalizability. One task was a choice reaction task (CRT) and the other was a memory recognition task (MRT). Each task was presented in both a spatial and verbal condition. No attempt was made to equalize the two types (CRT and MRT) of discrete tasks. The statistically significant main effect for both reaction time and error rate due to task type demonstrated that the MRT was more demanding than the CRT at both levels of difficulty. Also, there was a greater difference between easy and hard levels for the MRT than for the CRT as indicated by the statistically significant difficulty-by-task-type interaction for both measures of performance for the discrete task. There was a significant tracking presentation-by- task-type interaction for error rate. A post-hoc Tukey test revealed that this interaction was due to no performance difference between the single and dual task conditions, and no performance differences as a result of increasing the difficulty level of tracking. Based on the error rate data, it might be interpreted that the CRT task did not increase the demand for resources. However, there was a dual task decrement in reaction times for the CRT. Although there was no tracking-presentation-by-task-type interaction for reaction times the trend was the same for the CRT and MRT: both tasks demonstrated a single-to- dual task decrement but performance was not significantly affected by the increasing level of difficulty of the second task. The tracking task performance was not affected by the task type in any situation: There was no main effect and no interactions with task type.

Since an attempt was made in the preliminary study to equate the verbal and spatial task, as expected there was no statistically significant main effect due to resources, thus confirming that the spatial and verbal tasks were equal with regard to reaction times. However, there was an effect of error rate due to resources. A speed-accuracy trade-off is generally indicated by an inverse relationship between reaction time and error rate. As error rate increased, the reaction 
time did not decrease. Since the subjects held their reaction times constant and there was no significant interaction of difficulty with resource for the error rate, it can be concluded that there was no speed-accuracy trade-off in this experiment. The resource-by-task interaction for error rates indicates that the MRT which required spatial resources generated more errors than the verbal MRT. There was no such interaction for reaction time. As mentioned earlier, the items for the tasks were chosen based on reaction times and did not take into account the error rate. Items which were consistently missed in the preliminary study should have been deleted. This may have made the results more interpretable in that the resources would have been matched on both dependent measures of reaction time and error rate.

In order to support the multiple resource model a difficulty-by-resource interaction was expected (Navon \& Gopher, 1979). These interactions should result from a greater difference between easy and hard levels for the spatial task than for the verbal task in the dual task condition. There was no such interaction for elther the error rate data or tracking performance data. There was, however, a significant interaction for reaction time, but it was not in the expected direction of the spatial tasks having longer reaction times due to a competitlon for resources. Instead a post-hoc Tukey test revealed that this interaction was due to the fact that the more difficult level of the verbal task, in the dual task condition, yielded a longer reaction time than that of the hard spatial task: the difference in performance between easy and hard difficulty levels was greater for the verbal task than the spatial task. This is contrary to what was expected if the results supported the multiple resource model.

There was a statistically significant effect due to single versus dual task performance on both measures of reaction time and error rate. The difficulty-by-resource-by-task-presentation interaction indicated that neither resource, spatial nor verbal, was differentially affected as the 
difficulty of the tracking task increased. The tracking task performance was also not affected by either the difficulty or the resource of the discrete task.

As demonstrated by the difficulty-by-resource-by-task-presentation interaction for the reaction times there was no single-to-dual task difference in the hard spatial task (see Figure 9). This could indicate that the task was generally too difficult and subjects needed more time to discern some of the subtle differences between the stimulus and probe for this task (see Appendix E). Although the difficulty by resource-by-task-presentation interaction was not significant for error rate, the pattern was the same as that for the reaction time data: there was no significant difference between single and dual task performance for the hard spatial task. In spite of the extra time subjects used to respond to the MRT, their error rate remained high for the difficult spatial condition. According to the multiple resource model, the spatial discrete task performance should have been more highly impacted than the verbal task when paired with tracking. These results do not seem to support the multiple resource hypothesis, since it would be expected that the sharing of spatial resources by tracking and the spatial discrete task would show a performance decrement on either or both tasks. Generally, this did not occur. However, the error rate for the MRT for the hard difficulty level of the spatial resources was significantly greater than for all the other conditions. This interference apparently occurred as evidenced by the error rate data for the spatial MRT. Examination of the difficulty-by- resource interaction for reaction time indicated the effect was due to the hard verbal task (see Figure 7). Although the increased error rate in the dual task for the spatial condition could indicate support for the the multiple resource model, it is more likely due to the poor choice of items for the hard spatial MRT. These results do not necessarily support the multiple resource model, but might support a single pool resource model. There was a single-to-dual task performance decrement but neither 
combination of resources had a greater impact on the performance of either the discrete tasks or the tracking task (Kantowitz \& Knight, 1976).

The tracking task trials were divided into segments during which there were commanded changes in either zero, one, two, or all three axes (heading, speed, and altitude). As expected, the significant main effects due to segment for the RMSE on heading, altitude, and speed indicated that performance was affected as the related axis changed. The only axis which had a noise-by- segment interaction was heading. This result indicates that altitude changes affected the heading RMSE in the high noise level but not in the low noise level.

\section{Component and Overall Workload Ratings}

Three separate analyses were performed to analyze the workload ratings for: (a) the task components; (b) the whole task; and (c) the control condition of single task tracking compared with the dual task condition. The CWL ratings yielded three significant main effects and no significant interactions. The significant main effects were difficulty of the discrete task, tracking presentation, and task (only for the discrete task ratings and not for the tracking task ratings). There were only three effects which were significant for the overall workload (OWL) ratings; tracking presentation; difficulty by tracking presentation; and single-to-dual tracking performance. There was also a significant single-to-dual task main effect for tracking task performance. Both the CWL and OWL ratings indicated a significant single-to-dual task effect as indicated by the task presentation manipulation for the discrete task. Ratings were higher in the cuall task condition.

Hart (personal communication, November, 1988) suggested that the component workload ratings can be expected to follow certain patterns based on several different hypotheses. The first is the "consistency hypothesis" which states that ratings of components will not vary 
regardless of the demands imposed by concurrent activities. The subjects will not include time-sharing as part of their incurred workload.

The second hypothesis has two possible sub-pattems, one is directly related to the multiple resource model and the other deals with dissociation. In order to find evidence in support of the multiple resource model, the component workload ratings for the spatial task would be higher than those for the verbal task in the dual task condition. Although, according to Wickens (personal communication, November, 1987) when rating the components of the complex task, subjects will not be sensitive to resource competition due to the phenomenon referred to as dissociation. The theory of dissociation (Vidulich \& Wickens, 1986) states that the performance and workload measures will not mirror each other since a different cognitive process is used to determine the subjective workload ratings on a task from the process required to peiform the task.

The third hypothesis is also based on a limited capacity model but refers to a single pool of resources. This hypothesis states that the configuration would follow the multiple resource model, in that there will be a single-to-dual task performance decrement, but there would be no effect on component or overall ratings due to resources.

The CWL ratings were affected by the difficulty of the discrete task whereas the OWL ratings were not. These results demonstrale that subjects were sensitive to the difficulty manipulations of the discrete task components. The subjects did not rate the OWL significantly higher when the discrete task component of the complex task increased in difficulty. Although not statistically significant, the OWL ratings did increase as the discrete task demands increased. This demonstrates that component workload ratings as compared to OWL ratings could provide a more accurate account of the subjects' perceived workload. 
The significant main effect for component ratings due to tracking presentation indicates that when tracking is added to the discrete task, the CWL ratings increase. The component ratings of the discrete task also increased as the difficulty level of tracking increased. These results failed to support the hypothesis stating that workload ratings would be the same for components of a task, whether those components are isolated or paired. The component ratings for the discrete tasks increased in the dual task condition compared to the single task condition. This relationship was also shown in the low to high noise comparison: the CWL increased as the noise level increased. These results do not support the consistency hypothesis in that the ratings of the discrete task should have remained stable regardless of the demands imposed by concurrent activities.

Gopher and Donchin (1986) concluded that as difficulty increases subjects can maintain their performance because of an increase in effort demonstrated by an increase in their perceived workload ratings. The main effect due to tracking presentation on OWL may support this construct in that subjects increased their effort on the whole task in order to maintain consistent performance when the discrete task was paired with the tracking task. The OWL ratings were significantly affected by all three levels of tracking presentation; the single-to-dual task workload ratings increased as the difficulty of tracking increased. Kahneman (1973) might claim that these results indicate subjects increased their effort and thus increased their avaliable capacity.

The OWL ratings showed an effect for the single-to-dual task manipulation as well as for the easy to hard tracking manipulation. In the dual task condition the subjects maintained their performance on the discrete tasks while tracking but as the difficulty of tracking increased, their tracking performance decreased and their OWL ratings increased. This result indicates that as any component of a complex task increases in task demand, the OWL ratings increase. 
However, when the difficulty of the discrete task increased, the OWL ratings did not increase. Since there was a significant effect for the component ratings of the discrete task due to difficulty it would be expected that the overall ratings would reflect the change in difficulty, since the consistency hypothesis is not supported. The reason for this finding could be that the tracking task which was the continuous task was more compelling, subjects' OWL ratings may be based on the more salient characteristics of the complex task.

The significant main effect for task type demonstrated that subjects workload ratings of the components of the complex task were consistent with the performance data. Subjects rated the MRT higher than the CRT. As indicated by the performance data for both reaction time and error rate, the MRT was a more demanding task than the CRT.

There was no signiticant interaction for tracking presentation by task although the trend of the increasing ratings as task demand increased was the same for the choice reaction task and the memory recognition task. The performance data had significant interactions for tracking presentation by task. This pattern was the same for the reaction time data for the MRT and CRT and the error rate data for the MRT. The CRT error rate was equal tor all three levels of tracking presentation.

In this experiment there was no significant main effect for component ratings due to resources. If dissociation is defined as subject insensitivity to resource competition then these results could support dissociation. However, support for dissociation requires that the multiple resource model is supported by the performance data. This was not the case. Both performance measures (reaction time and error rate), component workload ratings, and overall workload ratings seem to support a single pool resource model. This is demonstrated by the fact that as the task demand increased, performance decremented and workload ratings increased. 
Since the subjects' ratings increased as difficulty of the tracking task increased it can be concluded that the cost of time-sharing is included in the subjective workload ratings.

\section{Summary and Conclusions}

Generally, the performance measures of reaction time and error rate do not support a multiple resource model of competition for same resources but rather a single undifferentiated pool capacity model. Direct evidence for an undifferentiated pool of resources comes from the observation that the unrelated (discrete and tracking) concurrent tasks did interfere with

performance. The spatial discrete task did not demonstrate a different pattern of performance decrement from the verbal discrete task, whereas it would be expected that performance on the spatial task would be more greatly affected because it was competing for the same resource utilized by the tracking task.

Neither the component nor OWL ratings followed the expected pattern to support the multiple resource model. The workload ratings did not dissociate and were not affected by the resource manipulation; they followed basically the same pattern as the performance measures. As performance decreased, workload ratings increased.

Taking into consideration performance and workload measures, the results of this study seem to support a capacity model with a single undifferentiated pool of resources. These results imply parallel processing since the subjects were able to track and respond to the discrete tasks. Also, the results indicate that as task demands increase, mental activity or effort increases.

Component ratings appear to be more sensitive than the overall ratings, in that subjects' ratings reflected the increase in difficulty of the discrete task through the component ratings but not the OWL ratings. Also, as evidenced by the component ratings, subjects are able to rate components of a complex task, but include the concurrent cost of performing the second task. 


\section{Suggestions for Future Research.}

Although the tracking task is commonly used in examining divided attention, this experiment focused specifically on the central stage of information processing, wherəas most other research in this area has examined the input, output, and central processing stages. The tracking task may have had a greater effect in the response stage rather than the desired impact on the central processing stage. If this is true; there may not have been enough demand on the spatial resource in the central processing stage. The effect on resources due to error rate might have contounded the results; thus it would be important to re-examine the items used in the discrete spatial MRT. Also, the implementation of another continuous task, either spatial or verbal, which definitely utilizes central processing may produce more conclusive results. 
References

Allport, D., Antonis, B., \& Reynolds, P. (1972). On the division of attention: The disproof of the sirgle channel hypothesis. Quarterly Jeumal of Experimental Psychology. 24, 225-235.

Boles, D. B., \& Wickens, C. D. (1983). A comparison of homogeneous and heterogeneous display formats in information integration and nonintegration tasks. Office of Naval Research Technical Beport\# N-000-14-79-C-0658, 1-15.

Broadbent, D. (1958). Perception and communication. London: Pergamon.

Broadbent, D. (1982). Task and selective intake of information. Acta Psychologica, 50, 253-290.

Cherry, C. (1953). Some experiments on the reception of speech with one and two ears. Jeumal of Acoustic Seciety of America, 25, 975-979.

Deutsch, J., \& Deutsch, D. (1963). Attention: Some theoretical considerations. Psychological Beview, 70 , $80-90$

Gopher, D. \& Donchin, E. (1986). Workload-An examination of the concept. In K. Boff \& L. Kaufman (Eds.), Handbook of Perception and Human Performance (pp. 1-49). New York: Wiley \& Sons.

Hart, S. G., \& Staveland, L. E. (1988). Development of NASA-TLX (task load index): Results of empirical and theoretical research. In P. A. Hancock \& N. Meshkati (Eds.), Human Mental Workload (pp.239-250). Amsterdam, the Netherlands: North Holland.

Kahneman, D. (1973). Attention and effort. Englewood Cliffs, NJ: Prentice-Hall.

Kantowitz, B. H. (1988). Mental Workload. In P.A. Hancock (Ed.), Human Factors Psychology. Amsterdam, the Netherlands: North Holland.

Kantowitz, B. H., \& Knight, J. L. (1974). Test tapping and time-sharing. Joumal of Experimental Psychology, 103, 331-336.

Kantowitz, B. H., \& Knight, J. L. (1976a). Test tapping and timesharing, II: Auditory secondary task. Acta Psychologica, 40, 343-362.

Kantowitz, B. H., \& Knight, J. L. (1976b). On experimenter-limited processes. Psychological Review, 83, 502-507.

Kinsboume, M. (1981). Single channel theory. In D. Holding (Ed.), Human Skills (pp. 65-89). New York: John Wiley \& Sons, Ltd. 
McLeod, P. (1977). A dual task response modality effect: Support for multi-processor models of attention. Quarterly Joumal of Experimental Psychology, 29, 651-667.

Moray, N. (1959). Attention in dichotic listening: affective cues and the influence of instructions. Quarterly Journal of Experimental Psychology, 9, 56-60.

Moray, N., \& Jordan, A. (1966). Practice and compatibility in 2-channel short-term memory.

Psychonomic Science, 4, 427-428.

Moray, N. (1967). Where is capacity limited? A survey and a model. Acta Psychologica, 27, 84-92.

Moray, N. (1969). Attention: Selective processing in vision and hearing. London: Hutchinson Educational Ltd.

National Aeronautics and Space Administration. (1986). NASA workload consultant for field evaluation (wC Fielde): Computerized Version. Moffett Field, CA: NASA Ames Research Center.

Navon, D., \& Gopher, D. (1979). On the economy of the human-processing system. Psychological Review, 86, 214-255.

Ninio, A., \& Kahneman, D. (1974). Reaction time in focused and in divided attention. Joumal of Experimental Psychology, 103, 394-399.

Norman, D. A. \& Bobrow, D. G. (1975). On data-limited and resc:urce-limited processes. Cognitive Psychology, Z, 44-64.

Treisman, A. (1960). Contextual cues in selective listening. Quarterly Journal of Experimental Psychology, 12, 242-248.

Treisman, A. (1964a). Selective attention in man. British Medical Bulletin, 20, 12-16.

Treisman, A. (1964b). The effect of irrelevant material on the efficiency of selective listening. American Joumal of Psychology, 77, 533-546.

Treisman, A. (1969). Strategies and models of selective attention. Psychological Review, 76, 282-299.

Treisman, A. (1970). Perception and recall of simultaneous speech stimull. Acta Psychologica, 33, $132-148$.

Treisman, A. (1971). Shifting attention between the ears. Quarterly Joumal of Experimental Psychology, 23, 157-167.

Treisman, A., \& Davies, A. (1972). Divided attention to ear and eye. In R. Kornblum, (Ed.), Attention and Perfomance IV (pp. 101-117). New York: Academic Press. 
Vidulich, M. A., \& Wickens, C. D. (1981). Time-sharing manual control and memory search: The joint effects of input and output modality competition, priorities and control order. University of lllinois Engineering-Psychology Laboratory Technical Report EPL81-4/ONR-81-4)

Vidulich, M. A. \& Wickens, C. D. (1986). Causes of dissociation between subjective workload measures and performance: Caveats for the use of subjective assessments. Applied Eroonomics, 17, 291-296.

Wickens, C.D. (1980). The structure of attentional resources. In R. Nickerson (Ed.), Attentionand Performance VIII (pp. 297-316). Hillside, NJ: Erlbaum.

Wickens, C. D. (1984a). Processing resources in Attention. In R. Parasuraman \& D.R. Davies (Eds.), Varieties of Attention (pp. 53- 102). New York: Academic Press.

Wickens, C. D. (1984b). Engineering Psychology and Human Performance. Columbus Ohio: Charles E. Merrill Publishing Company.

Wickens, C.D. \& Liu, Y. (1988). Codes and modalities in multiple resources: A success and a qualification. Human Factors, $30,599-616$.

Wickens, C.D. \& Yeh, Y. (1986). A multiple resource modei of workload prediction and assessment. Proceedings of the IEEE International Conference on Systems. Man, and Cybernetics. New York: Institute of Electrical and Electronics Engineers. 

Task ID:

\section{RATING SHEET FOR OVERALL WORKLOAD}

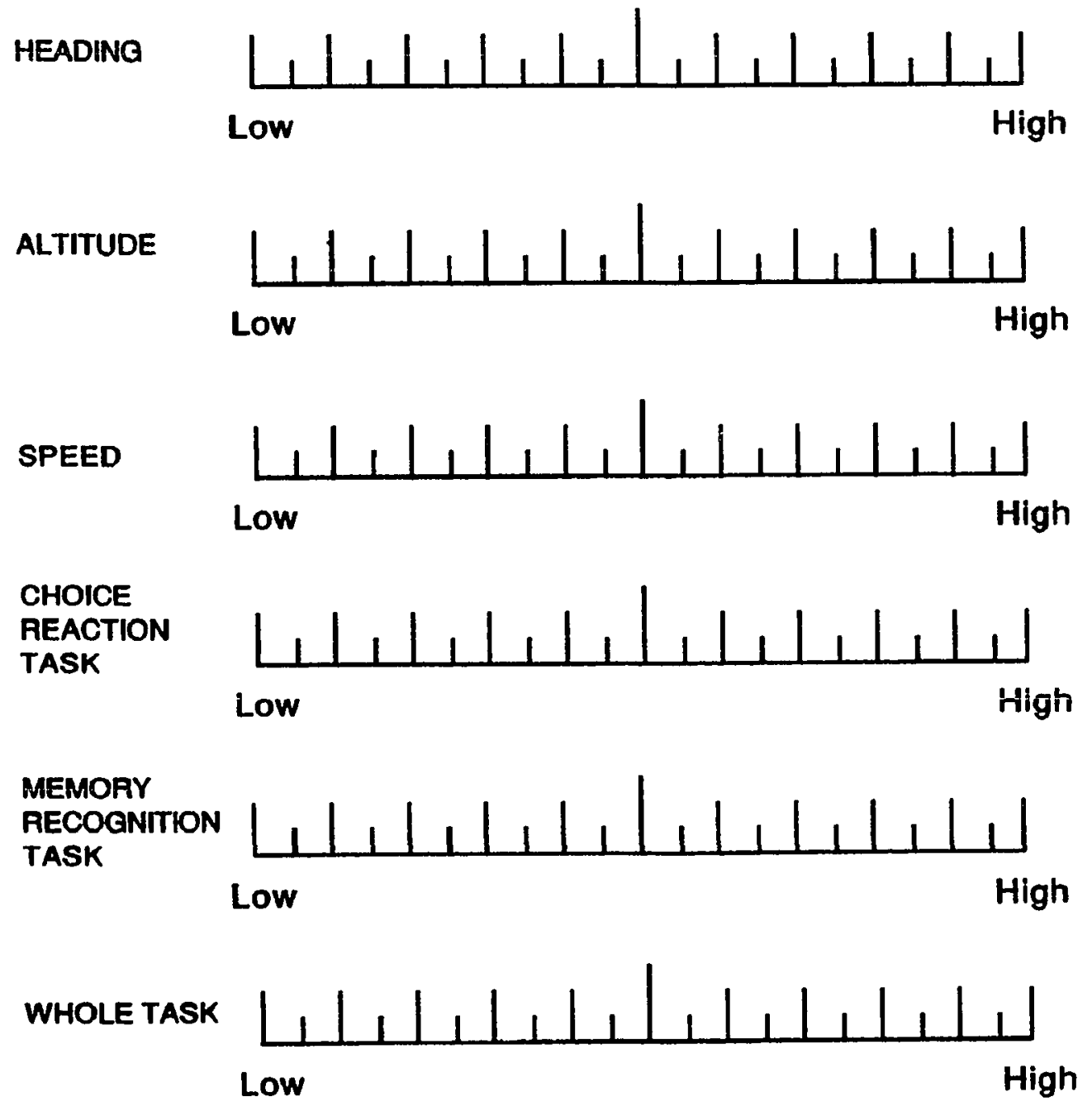


Appendix B

Easy Spatial MRT Stimuli

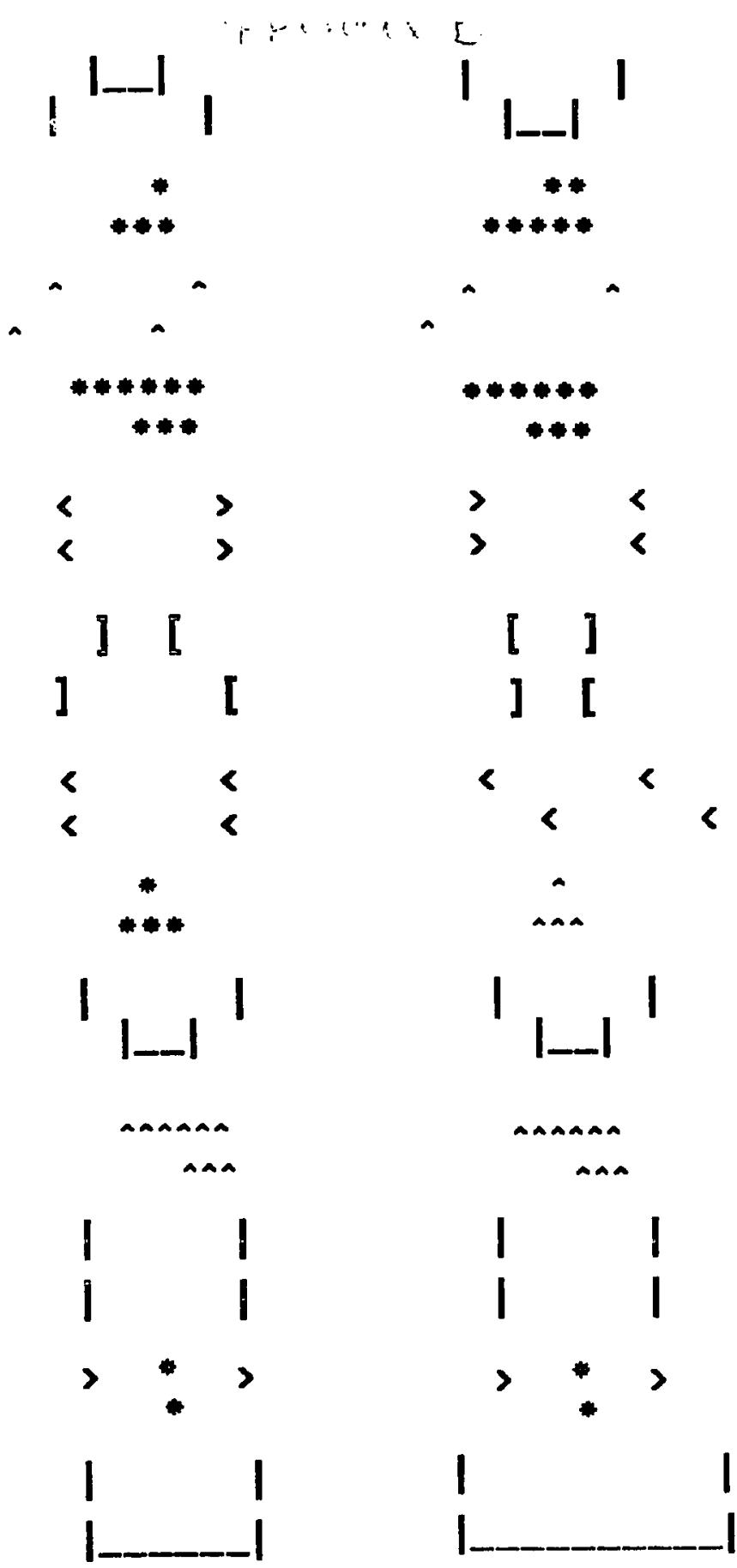




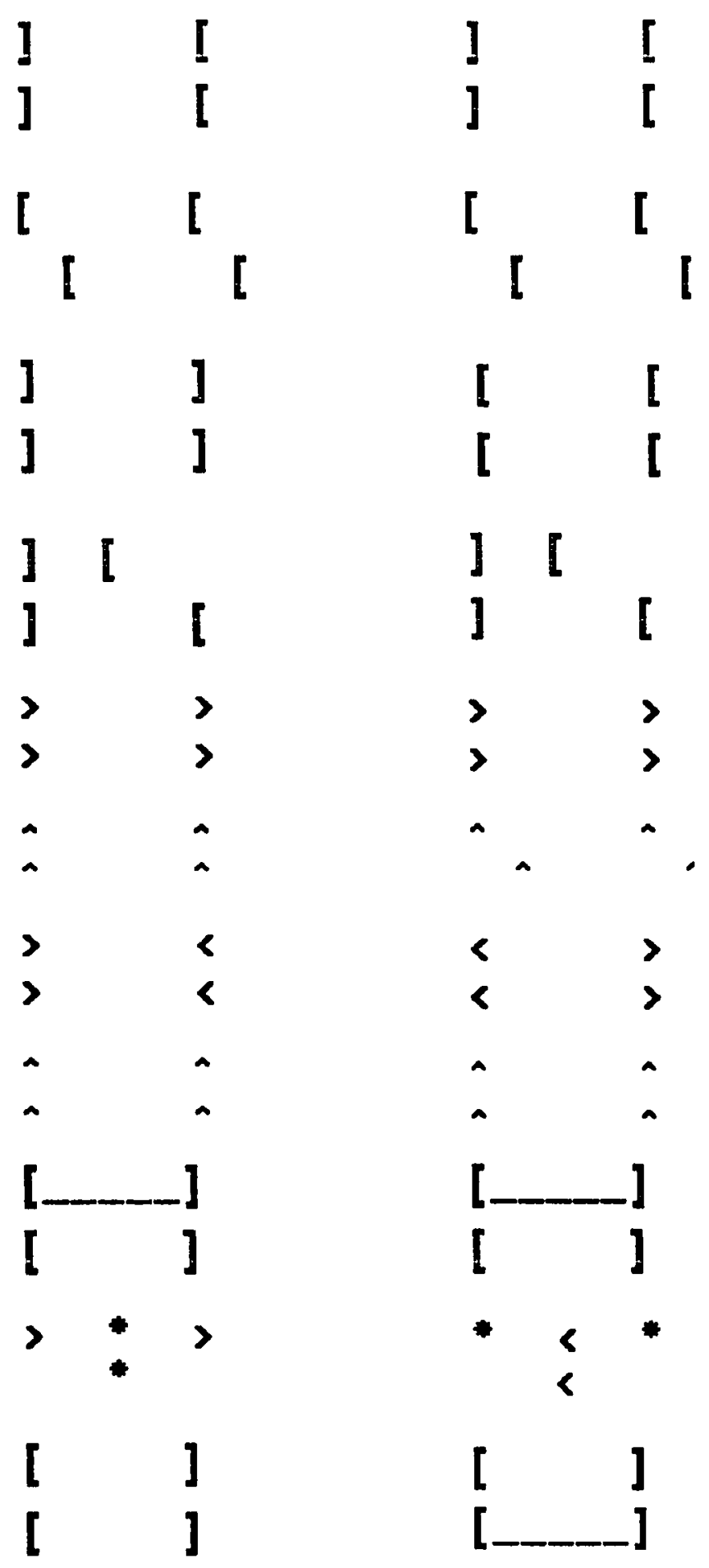


Hard Spatial Memory Recognition Task Stimuli
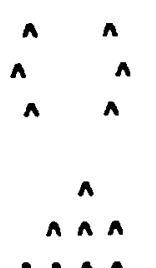

คคค $\wedge$

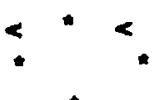

$<\cdots$
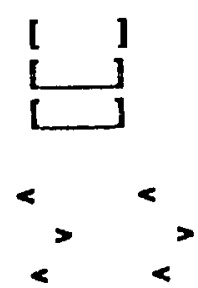

$\wedge \cap \wedge \cap \wedge ค \wedge$ $\wedge \cap \wedge \wedge$

ก $\cap$
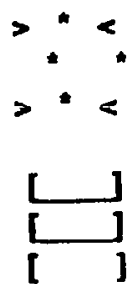

A

คคค

คคคคคคคค

$\wedge \wedge \wedge \wedge$

$\wedge \wedge$
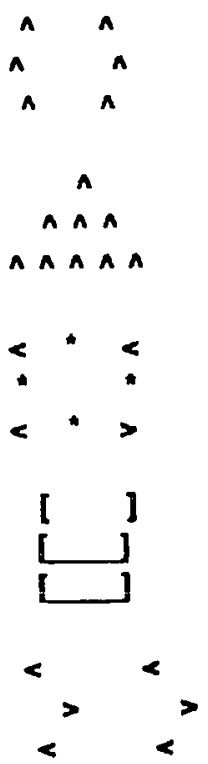

$\wedge \wedge \wedge \wedge \wedge \wedge \wedge$

$\wedge \wedge \wedge \wedge$

คค
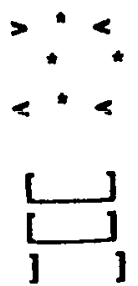

n

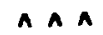

$\wedge \wedge \wedge \wedge$

คคคคคคคค

$\wedge \cap \wedge A$ 

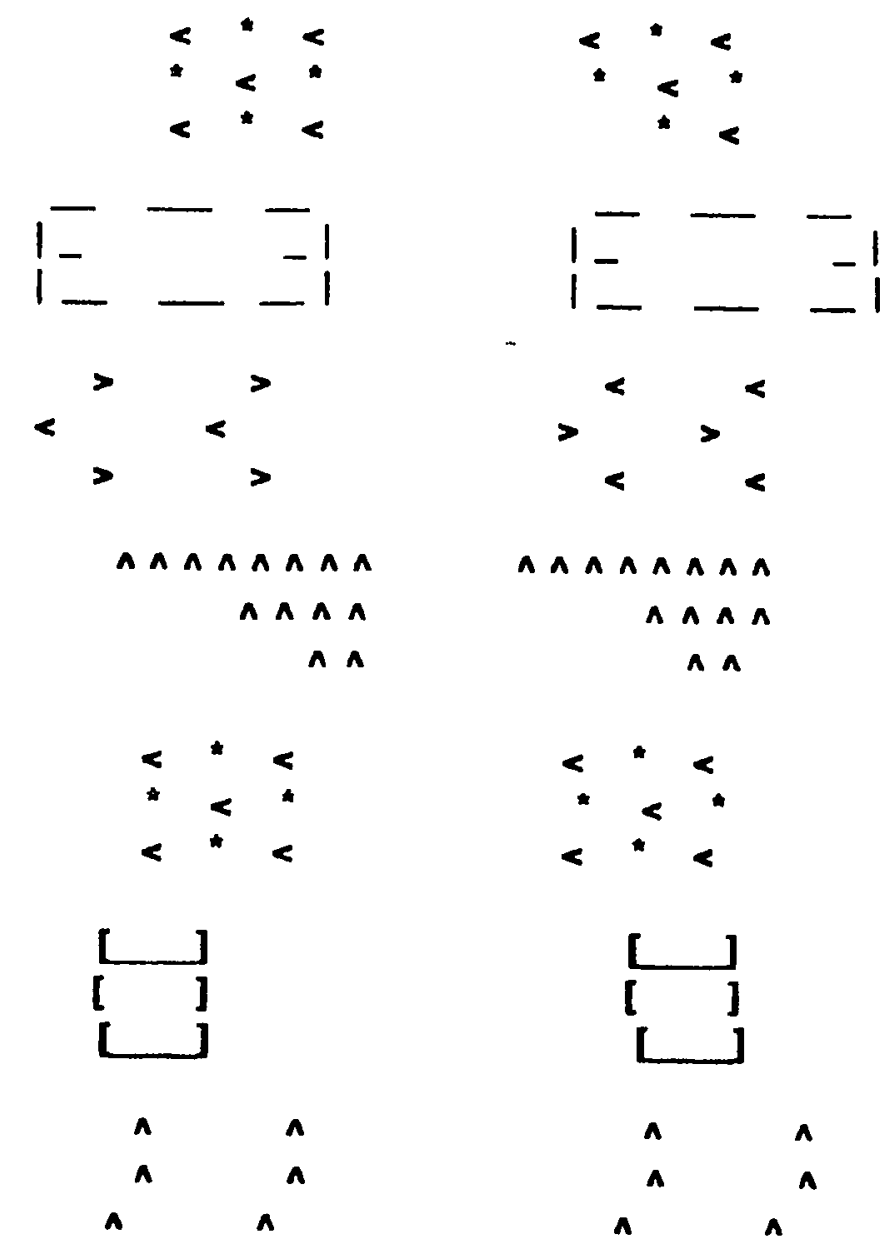

คヘヘヘヘヘヘ

คคค ค

ก
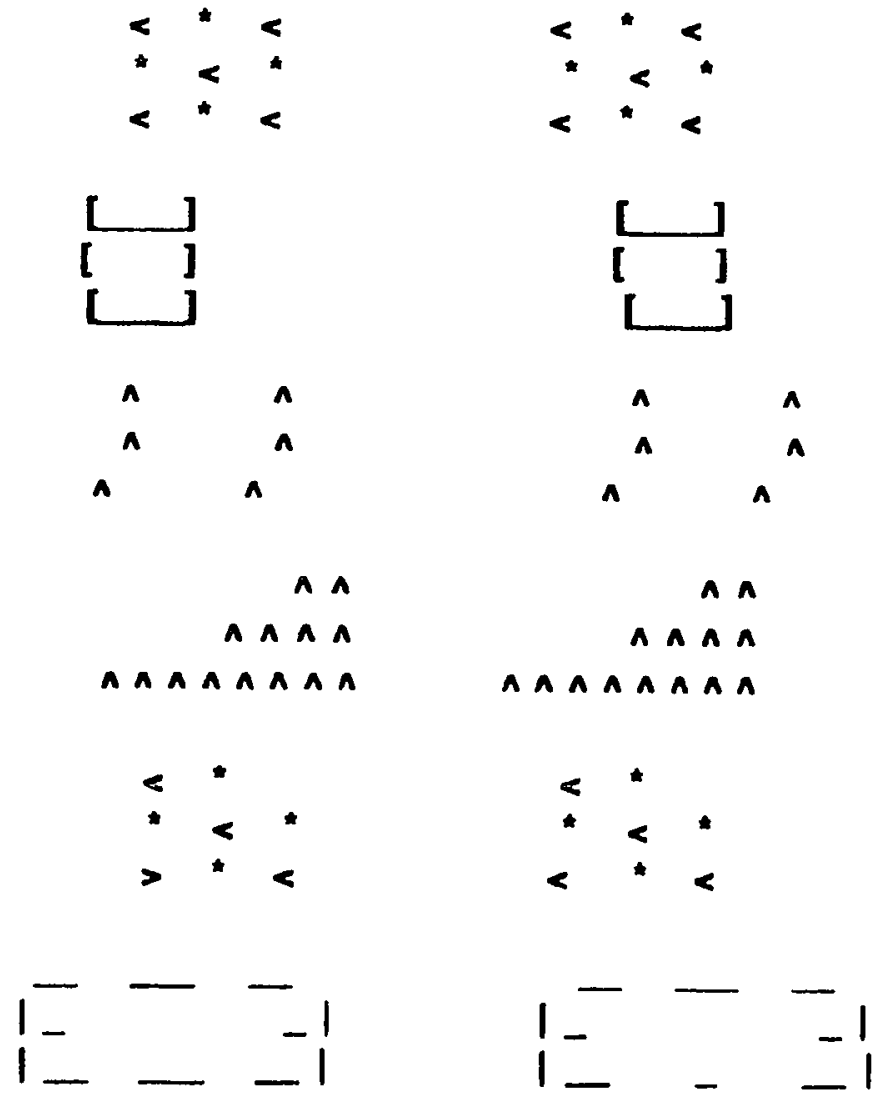

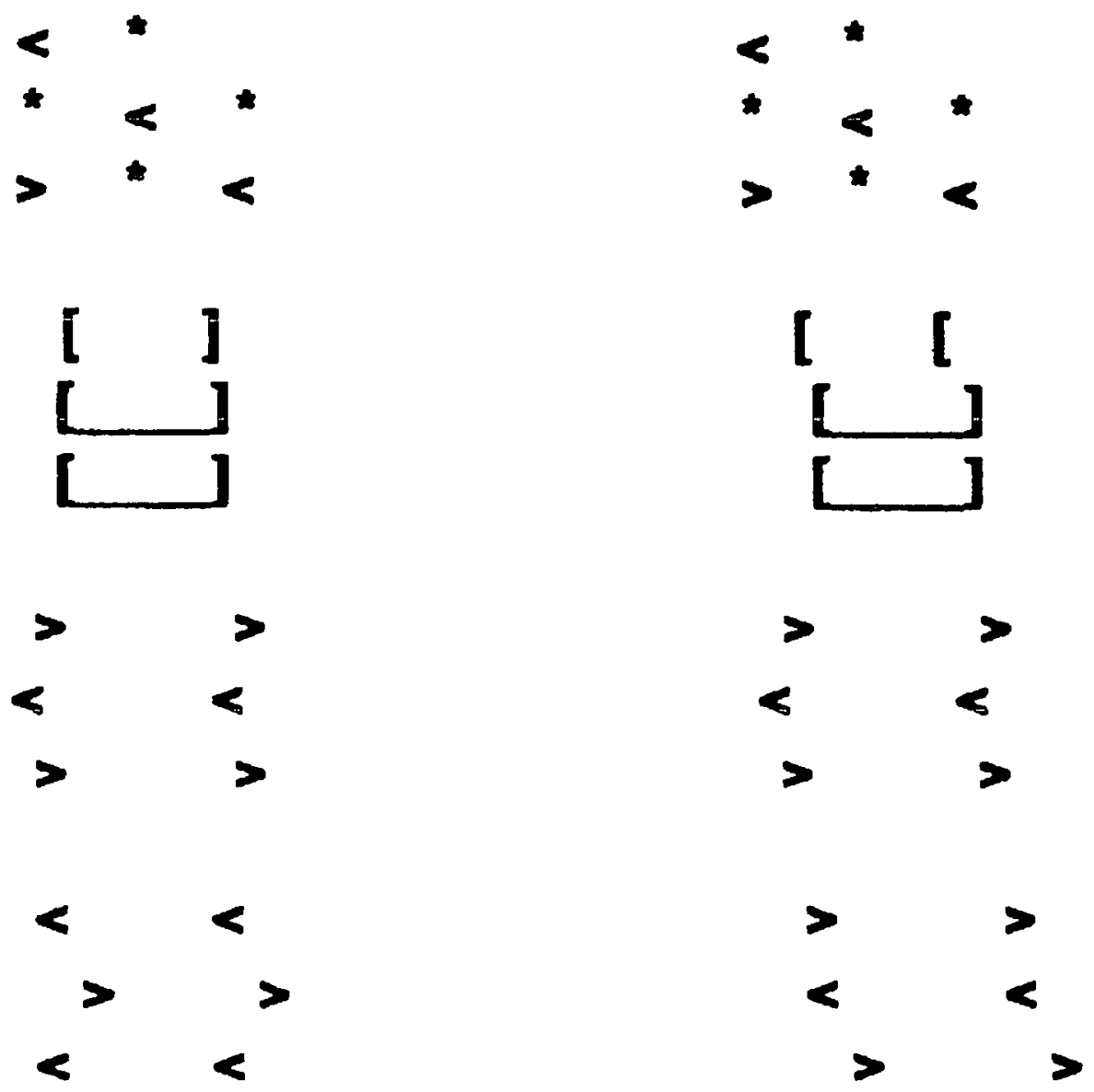


\author{
Appendix C \\ Hard Verbal Memory Recognition Task Stimuli
}

\begin{tabular}{|c|c|}
\hline PQ506P & PQ506P \\
\hline PQ640P & PQ640P \\
\hline QP506P & QP506P \\
\hline PQ460P & PQ460P \\
\hline SP506Q & SP506Q \\
\hline QP605S & QP605S \\
\hline PS506Q & PS506Q \\
\hline QP650S & QP650S \\
\hline SP506P" & SP506P \\
\hline RS506S & RS506S \\
\hline PP506S & PP506S \\
\hline RS560S & RS560S \\
\hline QP405R & QP405R \\
\hline PR506S & PR506S \\
\hline QR405P & QR405P \\
\hline PS506R & PS506R \\
\hline RQ506S & RQ506S \\
\hline RS506Q & RS506Q \\
\hline
\end{tabular}


Appendix D

\section{INSTRUCTIONS TO SUBJECTS}

We are interested in finding out how people divide their attention when performing complex tasks. For the next couple of hours we will be teaching you to perform a particular complex task, which is made up of several simple tasks. We will measure your performance on each component of the complex task and we will asik you questions about how hard you feel you were working during the task.

The task which we will present to you is similar to flying a light aircraft. A pilot must attend to many things while he is controlling the aircraft. We will present a varlety of tasks to you which you will need to respond to while you are controlling the aircraft.

You will je seated in front of a computer monitor. The screen is divided into four sections. Different aspects of the complex task will be performed by paying attention to each of the four sections of the display. The aircraft is controlled by following a moving target which is presented in the upper right-hand portion of the screen. Your aircraft is shown by a solid circle with three points. The target that you must follow is shown by an unfilled circle. Your aircraft is always following the target circle and the control inputs you make should be to follow the target circle as closely as possible. For instance, if the target circle is to the left of your aircraft symbol, you must turn your aircraft to the left in order to minimize the distance between your aircraft and the target. Altitude and heading of your aircraft are controlled with the joystick. Speed of your aircraft is controlled with the vertical slide pot. In order to turn your aircraft to the right, you simply push the joystick to the right. To turn your aircraft to the left, you push the joystick to the left. In order to increase your altitude, you must pull back on the joystick (pull it toward you), and in order to decrease your altitude, you must push forward on the joystick (push it away from you). These 
control movements are similar to those required to control an aircraft. In order to increase altitude, the nose of the aircraft must be pointed up, and this requires the pilot to pull back on the stick.

In order to increase your speed, you must push the slide pot knob up. To decrease speed, you push the slide pot knob down. The range of speed values on the slide pot is 0 knots (at the bottom) to $160 \mathrm{knots}$ (at the top). The speed of your aircraft relative to that of the target is presented by varying the size and color of the target. When the target is larger than your aircraft, you are following too closely (going too fast) and must decrease your speed. The target will be displayed in blue when your speed is too great. When the target is smaller than your aircraft, you are too far behind the target (going too slowly) and you must increase your speed to catch up. The target will be displayed in red when your speed is too slow. When the speed of your aircraft is the same as the speed of the target, the target will be the same size as your aircraft and will be displayed in white. However, if the target is speeding up and the slide pot is in the centered position, the distance between your aircraft and the target will increase. Each position on the slide pot maintains a particular speed. If, for instance, you move the slide pot knob to the center of the slide pot, you aircraft will increase its speed until it reaches 80 knots.

For all of these aircraft controls, it is best to provide small inputs and wait to see the effect. Large movements may result in overcorrections.

In addition to the lilustration of your aircraft symbol and the target symbol, digital presentation of information will also be presented in the upper right-hand section of the screen. You can see your aircraft's current altitude, heading, and speed.

In the upper left-hand section of the screen, several different messages will be presented. Most of these messages will require a response from you. There are five different types of 
messages which will be displayed. These five types of messages may appear at any time while you are flying, and they may appear in any order.

The control box which has the joystick and the slidepot also has several buttons. These buttons are labelled "YES", "NO", and "1", $2^{n}{ }^{n} 3^{n}$, and "4". You will use these buttons to respond to the tasks which will be presented in the message window while you are controlling the aircraft. The use of these buttons will be described to you as we describe each of the other tasks.

I will now briefly describe the two different types of tasks that you will be performing while you are flying the aircraft. One type will be a memory recall type of task and the other will be a four choice position indicator task. You will be given additional instructions and specific examples of each type of task before each trial.

General Information concerning tasks in the message window Memory recall task (MRT)

You will see either a 4 or 7 digit alphanumeric message in the message window, you are to retain this message in your memory and decide whether or not the following message is the same. In another type of memory task you will see either a $2 \times 2$ or $3 \times 3$ array of nonalphanumeric characters and will se expected to decide whether the following message is the same. If the answer is the same you will press button "yes" if the answer is different you will press button "no".

Another type of task that you will be required to perform while flying is a four-choice position indicator task. Four X's will be displayed in the upper left hand portion of the display, and beneath these four X's will be one $X$. These X's correspond to the four buttons at the bottom of the control box. You should press the button which corresponds in position to the single $X$ below 
the four $X$ 's. You will also see the digits 1 through 4 with an $X$ pointer, in this case you will press the button number which the $X$ is pointing to.

In another aspect of the above task you will see one or two arrows ( ) above the row of X's the direction and number of arrows will require you to press the button to the one or two to the left or right of the pointer. When the digits are presented you may see $a+$ or -1 or 2 , you will be required to observe the number indicated by the pointer, mentally perform the calculation and press the resulting button number.

You should perform each of these tasks as quickly as you can while continuing to fly the aircraft and follow your target as closely as possible in heading, altitude, and speed. At the end of each flight (about 8 minutes) we will show you how well you performed each of the tasks. At times, you will be asked to fly without answering any questions, and at other times you will be asked to answer questions without flying. We will also ask you to rate how difficult different aspects of the task were for you to do. We will give you separate instructions about how to rate your workload.

Please tell us if you have any questions that we can clarify for you. There will be demonstration trials for you to become familiar with the requirements for this experiment before we start collecting data.

Before the start of the experiment you will receive a demonstartion and any questions will be answered. 
Appendix E

CHANGES IN FLIGHT TASK BY SEGMENT

\begin{tabular}{|c|c|c|c|c|c|c|c|c|}
\hline AXIS & S1 & S2 & S3 & S4 & S5 & S6 & S7 & S8 \\
\hline SPEED & -- & $x$ & - & -- & $x$ & $x$ & -- & $x$ \\
\hline HEADING & -- & -- & $x$ & -- & $x$ & -- & $x$ & $x$ \\
\hline ALTITUDE & -- & -- & -- & $x$ & - & $x$ & $x$ & $x$ \\
\hline $\begin{array}{l}\text { ORDER IN } \\
\text { SCENARIO }\end{array}$ & 6 & 1 & 5 & 2 & 8 & 4 & 3 & 7 \\
\hline
\end{tabular}

$X=$ CHANGE IN TRACKING

$--=$ NO CHANGE IN TRACKING 